\title{
A theory of dispersion in a porous medium
}

\author{
By P. G. SAFFMAN \\ Cavendish Laboratory, University of Cambridge
}

(Received 16 January 1959)

This paper is concerned with the dispersion of a dynamically neutral material quantity in a fluid flowing through a porous medium. The medium is regarded as an assemblage of randomly orientated straight pores, and it is assumed that the path of a marked element of the material quantity consists of a sequence of statistically independent steps whose direction and duration vary in some random manner. The probability density function for the displacement of a single marked element is calculated and values for the dispersion of a cloud of marked elements then follow.

The case is examined in which the flow satisfies Darcy's law (i.e. the mean velocity is linearly proportional to the mean pressure gradient), and the molecular diffusivity is sufficiently small for the dispersion to be primarily due to the randomness of the streamlines, but it is not assumed that effects of molecular diffusion can be altogether neglected. It is shown that the longitudinal dispersion in the direction of the mean flow may be described asymptotically by an effective diffusivity which is a function of $U, l, a, \kappa$ and $T$. ( $U$ denotes the average velocity, $l$ the pore length, $a$ the pore radius which is shown to be related to the permeability, $\kappa$ the molecular diffusivity, and $T$ the time from the initial instant.) Expressions for the longitudinal diffusivity $\kappa_{l}$ are obtained according to the relative values of $l / U, T, t_{0}=l^{2} / 2 \kappa$ and $t_{1}=a^{2} / 8 \kappa$. These are given in $\S 4$, equations (4.3), (4.4) and (4.5). Speaking roughly, when $t_{0} \gg T \gg l / U, \kappa_{l} / U l$ is a logarithmic function of $U T / l$ and increases with $T$; when $T \gg t_{0} \gg l / U$, which must eventually be the case however small $\kappa, \kappa_{l} / U l$ is a logarithmic function of $U l / \kappa$ and independent of $T$. The theoretical results are compared with experimental data reported in the literature and approximate agreement is obtained when $l$ is put equal to the average diameter of the particles composing the porous medium.

The lateral dispersion in the direction perpendicular to that of the mean velocity is found to be governed asymptotically by an effective diffusivity $\kappa_{t}=\frac{3}{16} U l$. However, it is pointed out that some of the assumptions, namely that successive steps are statistically independent and that the dispersion of a cloud follows immediately from the statistical properties of the displacement of a single marked element, may not be valid for the lateral dispersion and this result is therefore suspect.

Remarks are made in $\$ 5$ on the dispersion for high values of the Reynolds number $U l / \nu(\nu=$ kinematic viscosity) when Darcy's law is not obeyed, and it is argued that $\kappa_{l} / U l$ should decrease as $U l / \nu$ increases. 


\section{Introduction}

When a viscous fluid flows through the pores and voids of a porous medium, such as a bed of sand, a tower of small glass beads, a porous rock like limestone, etc., a material quantity carried by the fluid (e.g. a substance in solution or heat) is dispersed by molecular diffusion and by what may be called 'convective' or 'mechanical diffusion'. The latter effect arises from the irregular pattern of the streamlines through the pores and voids, and the consequent tendency for fluid elements which are originally close together to become separated. The situation is in fact somewhat analogous to that of turbulent diffusion, the difference being that in a porous medium the irregularity of the streamlines arises from the complicated geometrical structure of the medium instead of from random solutions of the Navier-Stokes equations.

The purpose of this paper is to examine the 'convective diffusion' of a dynamically neutral material quantity or, as is equivalent, the phenomenon of 'miscible displacement' when a viscous fluid is expelled from a porous medium by another fluid of identical viscosity and density with which it is completely miscible, since in this case the relative concentrations of the fluids may be regarded as a dynamically neutral material quantity. In an actual flow further complications are often present; for example, adsorption of the material quantity on the solid material of the medium may occur, the physical properties of the fluid may vary significantly with the concentration of the material quantity, etc. Such effects are often of great practical importance, but we shall not consider them here at all.

The detailed structure of the medium is usually highly irregular and only a few statistical properties are in practice available. A detailed solution for the flow pattern is therefore not a practical possibility, and even if it were it is doubtful whether it would be useful because of its complexity. However, the quantities of interest are often spatial averages; for instance, the filter velocity which is the flux of fluid per unit area of the medium, the 'average' or interstitial velocity which is the spatial average of the velocity over the region occupied by fluid, ${ }^{*}$ and the mean pressure gradient and mean concentration of a material quantity which may be likewise defined. (It need hardly be pointed out that the spatial average is a useful concept only if the medium and the flow are effectively statistically homogeneous over a region large compared with the dimensions of the grains or

* The average velocity is in fact the filter velocity divided by the porosity (the fraction of porous media actually occupied by fluid), since the filter velocity is

$$
\int \mathbf{u} d \boldsymbol{S} / \int d \boldsymbol{S}=\int \mathbf{u} d V / \int d V
$$

where $d S$ and $d V$ denote elements of area and volume of the porous media and $\mathbf{u}$ is the velocity of the fluid. The average velocity is

$$
\int \mathbf{u} d V^{\prime} / \int d V^{\prime}=\int \mathbf{u} d V / \sigma \int d V,
$$

where $d V^{\prime}$ is an element of volume entirely occupied by fluid, since $\mathbf{u}=0$ if $d V \neq d V^{\prime}$ and by definition

where $\sigma$ denotes the porosity.

$$
\int d V^{\prime}=\sigma \int d V
$$


pores.) It seems reasonable to suppose that the various ways in which such averages may depend upon one another will not be greatly affected by changes in the detailed structure of the medium which leave its statistical properties unaltered, and that it will be profitable to consider not the flow through an actual porous medium but a simple model of such a flow, the model being constructed so that it appears to correspond in essentials with the actual flow, and is capable of mathematical analysis. Scheidegger (1957) gives an account of various models which have been put forward to calculate the permeability (i.e. resistance to flow) of porous media.

A 'random-residence-time' model for longitudinal dispersion has been put forward and developed by several writers (see, for example, McHenry \& Wilhelm 1957, where other references are given). (When the average velocity is unidirectional, the dispersion in the same direction is termed longitudinal or axial dispersion, and that in the perpendicular directions lateral or transverse dispersion; there is no reason why these should be equal even though the medium is statistically isotropic.) Briefly, this theory considers the flow through a sequence of cells in each of which there is complete mixing, i.e. in which the probability of a fluid element leaving a cell in any interval of time is independent of the length of time it has been in the cell. The time of passage of a fluid element through a length of the porous medium is then the sum of the times spent in each cell, and is a random function which tends to a Gaussian form with a variance proportional to the number of cells as this number becomes large. It can then be shown that the longitudinal dispersion is described by the diffusion equation with an effective diffusivity proportional to the product of the average velocity and the length of each cell. Identifying this length with the diameter of the solid particles of which the medium is composed, one obtains values in reasonable agreement with experimental observations at values of the Reynolds number, based on the average velocity and average particle size, in the range 50-200. For these Reynolds numbers, the flow in the voids may be turbulent, producing fairly intensive mixing, so that the model appears to be a not unreasonable simplification of the actual flow. However, it does not lend itself to a study of lateral dispersion, and also the validity of the model to describe what happens at low Reynolds numbers is open to question.

An alternative approach to the problem of dispersion is to follow the motion of a marked fluid particle, supposing that its path varies in some postulated random manner. The probability distribution of the displacement of a single fluid particle after a given time may then be calculated. If the distance traversed or the time taken is sufficiently large for the assumption to be valid that the paths of initially neighbouring particles become statistically independent, the dispersion of a marked volume of fluid can be obtained.

Several writers (e.g. Baron 1952; Scheidegger 1954) have investigated dispersion on the assumption that a fluid particle carries out a random walk consisting of a succession of statistically independent straight steps in equal small intervals of time. It follows from the Central Limit Theorem of statistics that the probability distribution function of the displacement is Gaussian with a variance proportional to the time, and hence that the dispersion is described by the 
diffusion equation with the effective diffusivity related to the parameters of the random walk.

This treatment is physically not entirely satisfactory, partly because the time is broken up into equal small intervals and it would be expected intuitively that a particle stays longer in a region where the velocity is small than where it is large, and also because it seems to predict that the dispersion is isotropic since there is no obvious mechanism whereby the velocity fluctuations about the mean, and thence the displacement about the mean position, are correlated with the direction of the average velocity.

The present work originated in a suggestion by Sir Geoffrey Taylor that the dispersion might be investigated by taking as a model of the flow through a porous medium that through an assembly of randomly orientated and distributed straight pores, in each of which the flow is uniform. The pores are supposed to be connected with one another at the ends and several pores may start or finish at these end-points. The dimensions of the pores are to be taken as comparable with the size of the particles composing the bed. In other words, the medium is visualized as a lattice in which a randomly distributed set of points are connected to their neighbours by straight uniform pores. The path of a fluid particle may then be regarded as a random walk in which the length, direction, and duration of each step are random variables. The probability distribution function of the displacement of a fluid particle after a given time may then be calculated and a value for the dispersion obtained.

One condition which must be satisfied in order that this model should apply to the actual dispersion of a material quantity is that the amount of dispersion that takes place is large compared with the dispersion there would be due to molecular diffusion acting alone as under static conditions, i.e. the effective diffusivity must be large compared with the molecular diffusivity. It will be assumed throughout this paper that this is the case. This is not to say, however, that molecular diffusion is entirely negligible; on the contrary, it will be found that the theory to be developed below predicts that in some cases the effective diffusivity is a logarithmic function of the molecular diffusivity (cf. Taylor $(1953,1954)$ where it is shown that the effective diffusivity describing the dispersion of a material quantity in a fluid flowing through a long capillary tube is inversely proportional to the molecular diffusivity). But it is expected that this theory is valid only if the molecular diffusivity is sufficiently small compared with the product of the average pore length and average velocity which, as will be seen, determines the order of magnitude of the effective diffusivity. It is worth mentioning in passing that there are physical circumstances of great interest (e.g. geothermal flows) in which 'convective diffusion' is small compared with molecular diffusion and may be entirely neglected.

After this paper had been completed, it was discovered that Josselin de Jong (1958) had considered a similar model and obtained one of the results of this paper, namely that of equation (4.4b) below. However, the model discussed in this paper is rather more general, and various results are obtained, that given by Josselin de Jong being a particular case apparently valid only for a certain limited range of the physical parameters. 


\section{Description of the model and formulation of the random walk}

In this section we describe the model for calculating dispersion in a porous medium which supposes that the medium is an assembly of randomly orientated straight uniform pores and that a marked particle moves along a random succession of straight steps, each step corresponding to passage through one pore, of variable direction and duration. It is supposed that the medium is statistically homogeneous and isotropic, and that the average velocity or mean pressure gradient is constant and unidirectional. We take a co-ordinate system with the $x$-axis in the direction of the average velocity, and denote by $l$ the length of a step, by $\theta$ the angle between the direction of motion along the step and that of the average velocity, by $\phi$ the azimuthal angle between the $y$-axis and the projection of the step on the $y z$-plane $(0 \leqslant \phi<2 \pi)$, by $t$ the duration of the step, and by $q=l / t$ the velocity of the marked particle along the step. Then the displacement of a marked particle after $n$ steps is a random variable with components parallel to the axes

$$
X_{n}=\sum_{1}^{n} l_{r} \cos \theta_{r}, \quad Y_{n}=\sum_{1}^{n} l_{r} \sin \theta_{r} \cos \phi_{r}, \quad Z_{n}=\sum_{1}^{n} l_{r} \sin \theta_{r} \sin \phi_{r}
$$

and the time for $n$ steps is the random variable

$$
T_{n}=\sum_{1}^{n} t_{r}=\sum_{1}^{n} l_{r} / q_{r}
$$

where the suffix $r$ refers to the $r$ th step. In order to obtain the probability distributions of the various random variables, we must now examine the flow through the model.

\section{The velocity of the fluid through the pores}

Let us consider the average speed, $\hat{q}$ say, of the fluid flowing through a particular pore, i.e. $\hat{q}$ is the flux of fluid through the pore divided by its cross-section area; it is in general a random quantity which varies from pore to pore. (It is called here the 'average speed' to distinguish it from the 'average velocity' which in this paper is a spatial mean over many pores.)

We shall now suppose that the inertia of the fluid is negligible and that the motion through the pores is dominated by viscosity. In this case the NavierStokes equations which describe the motion of the fluid through the pores become linear and the average velocity will be linearly proportional to the mean pressure gradient and inversely proportional to the viscosity of the fluid. That is, Darcy's law is satisfied according to which

$$
Q=k P^{\prime} / \mu
$$

where $Q$ is the filter velocity, $P^{\prime}$ is the mean pressure gradient, $\mu$ is the viscosity, and $k$ is a constant with dimensions (length) ${ }^{2}$ which is a property of the porous medium and is called the permeability. The range of validity of Darcy's law depends upon the Reynolds number of the motion through the voids and the structure of the medium (see Scheidegger 1957).

Further, the average speed $\hat{q}$ is proportional to the pressure drop between the ends of the pore divided by the length of the pore, i.e. to the pressure gradient 
along the pore. Now the pressure in the fluid is $P^{\prime} x+p$, where $p$ is a random quantity whose spatial average over many pores is zero, and if the fluid inertia is negligible it is not unreasonable to suppose that $p$ does not vary too rapidly with position and that the pressure gradient in a pore making an angle $\theta$ with the $x$-axis and with azimuthal angle $\phi$ is

$$
\begin{aligned}
\left(P^{\prime}+\frac{\partial p}{\partial x}\right) \cos \theta+ & \frac{\partial p}{\partial y} \sin \theta \cos \phi+\frac{\partial p}{\partial z} \sin \theta \sin \phi \\
& =P^{\prime}\left\{\left(1+p_{1}\right) \cos \theta+p_{2} \sin \theta \cos \phi+p_{3} \sin \theta \sin \phi\right\}
\end{aligned}
$$

where $\left(p_{1}, p_{2}, p_{3}\right)=\left(1 / P^{\prime}\right) \operatorname{grad} p$ are dimensionless random quantities with zero mean whose statistical properties depend entirely upon the medium and are independent of the magnitude of $\boldsymbol{P}$ (since the equations of motion are linear) and also of $\theta$ and $\phi$. Then,

$$
\hat{q}=\frac{A P^{\prime}}{\mu}\left\{\left(1+p_{1}\right) \cos \theta+p_{2} \sin \theta \cos \phi+p_{3} \sin \theta \sin \phi\right\},
$$

where $A$ is the cross-section area of the pore multiplied by some function of its shape. By definition, $\hat{q}$ is a positive quantity and the values of $\theta$ and $\phi$ for a pore (or step) are defined as those $(0 \leqslant \theta \leqslant \pi ; 0 \leqslant \phi<2 \pi)$ for which (2.5) is positive.

In general, $A$ will be a random quantity varying from pore to pore. However, we shall assume, primarily for the sake of simplicity, that all the pores are of circular cross-section and of equal radius $a$, say, so that $A=\frac{1}{8} a^{2}$. This assumption is physically not very good, but it actually involves no real loss of generality and does not affect the results significantly. It is perfectly possible to go through the analysis with $A$ as a random variable (and even with the cross-section of a pore varying), but since we do not know the statistical properties of $A$ and in any case the model is only an approximation to an actual porous medium, it seems hardly worth while introducing extra complications of this kind. For similar reasons, all the pores will be supposed to be of the same length $l$, say, where $a$ should be small compared with $l$ for the model to be strictly meaningful.

\section{The average velocity and permeability of the model}

The probability density function (p.d.f.) of $\left(p_{1}, p_{2}, p_{3},\right)$ will be supposed Gaussian and isotropic* so that the probability that it lies in the range $\left(p_{1}, p_{2}, p_{3}\right)$ to $\left(p_{1}+d p_{1}, p_{2}+d p_{2}, p_{3}+d p_{3}\right)$ is

$$
\left(\frac{\lambda}{\pi}\right)^{\frac{3}{2}} \exp \left[-\lambda\left(p_{1}^{2}+p_{2}^{2}+p_{3}^{2}\right)\right] d p_{1} d p_{2} d p_{3}
$$

where $\lambda$ is some dimensionless constant parameter which will depend only on the structure of the porous medium. The value of $\lambda$ is a measure of the relative magnitude of the mean pressure gradient and the fluctuations; $\left(p_{1}, p_{2}, p_{3}\right)=0$ corresponds with $\lambda=\infty$.

* The assumption that this p.d.f. is isotropic is unlikely to be strictly correct since there is in fact a preferred direction, namely that of the average velocity, but it considerably simplifies the analysis and is unlikely to lead to a significant error. 
The proportion of pores such that the values of $\theta, \phi, p_{1}, p_{2}$ and $p_{3}$ lie in the element of generalized volume $d \theta d \phi d p_{1} d p_{2} d p_{3}=d \Sigma$, say, is therefore proportional to the product of $\sin \theta d \theta d \phi$ and (2.6), and is

$$
\frac{1}{2 \pi} \sin \theta\left(\frac{\lambda}{\pi}\right)^{\frac{3}{2}} \exp \left[-\lambda\left(p_{1}^{2}+p_{2}^{2}+p_{3}^{2}\right)\right] d \theta d \phi d p_{1} d p_{2} d p_{3}
$$

where the domain of $\Sigma$ is $0 \leqslant \theta \leqslant \pi, 0 \leqslant \phi<2 \pi, \hat{q}>0$, and the constant of proportionality was chosen to make the integral of (2.7) over the domain $\Sigma$ equal to one, so that (2.7) is a true proportion.

The components parallel to the $x$-, $y$ - and $z$-axes of the average speed through a pore are

$$
\hat{q} \cos \theta, \quad \hat{q} \sin \theta \cos \phi, \quad \hat{q} \sin \theta \sin \phi,
$$

and, by definition, the components of the average velocity are the values of (2.8) averaged over many pores. The components of average speed in the $y$ - and $z$-directions clearly average to zero. The average velocity in the $x$-direction is denoted by $U$, where

$$
\begin{aligned}
U & =\int_{\Sigma} \hat{q} \cos \theta \frac{1}{2 \pi} \sin \theta\left(\frac{\lambda}{\pi}\right)^{\frac{3}{2}} \exp \left[-\lambda\left(p_{1}^{2}+p_{2}^{2}+p_{3}^{2}\right)\right] d \Sigma \\
& =\frac{a^{2} P^{\prime}}{24 \mu},
\end{aligned}
$$

on substituting from (2.5) for $\hat{q}$ and integrating (see the Appendix).

The filter velocity $Q$ is $\sigma U$, where $\sigma$ is the porosity and thus $Q=\left(\sigma a^{2} P^{\prime}\right) / 24 \mu$, whence the permeability $k=\left(\sigma a^{2}\right) / 24$. Note that $k$ is independent of $\lambda$. This expression may be used to obtain an estimate of $a$ which is otherwise very much an unknown quantity. For instance, Mr R. Wooding in the Cavendish Laboratory has measured the permeability of a bed of glass spheres of average diameter $2 \times 10^{-2} \mathrm{~cm}$ and obtained the value $k=3 \times 10^{-7} \mathrm{~cm}^{2}$, the value of the porosity being $0 \cdot 37$. Substituting into the formula for the permeability, we obtain $a \doteqdot 4 \times 10^{-3} \mathrm{~cm}$. Taking as a value for $l$ the average diameter of the spheres, we have $a / l=\frac{1}{5}$, which seems reasonable. The question of what value to give $\lambda$ will be deferred for a little while.

\section{The duration of the steps in the random walk}

We can now return to discuss the properties of the random walk. The velocity of an idealized fluid particle in a pore is

$$
q=2 \hat{q}\left(1-\frac{r^{2}}{a^{2}}\right)
$$

where $r$ is the distance of the fluid particle from the axis of the pore and the velocity profile has been assumed parabolic. The duration $t$ of a step by an idealized fluid particle would then be

$$
t=\frac{l}{q}=\frac{l}{2 \hat{q}\left(1-r^{2} / a^{2}\right)} .
$$

At first sight it might be thought that infinitesimal elements of the material quantity may be assumed to move through the pores like idealized fluid particles so that the duration of a step is given by (2.11). Speaking roughly, this is permissible if the duration of a step is small compared with the time required for 
effects of molecular diffusion to be appreciable. However, $t$ is large without bound for steps in which $r$ is nearly equal to $a$ or $\hat{q}$ is very small; so that even though the molecular diffusivity is as small as we please, there will be some elements of material quantity which are so long in a pore that molecular diffusion may be appreciable and it is not obvious that it is meaningful to regard them as moving like idealized fluid particles. We may intuitively expect this effect to be negligible if the proportion of idealized fluid particles undergoing steps of very long duration is sufficiently small, but exactly how small remains to be determined. To anticipate, it may be shown (see $\$ 3$ ) that the condition of being sufficiently small is that $\int_{0}^{\infty} t f(t) d t$ is finite, where $f(t) d t$ is the proportion of idealized fluid particles which at any instant are undergoing steps of duration in the range $t$ to $t+d t$. The proportion of pores in which the average speed is $\hat{q}$ is given by (2.7), the duration is given by (2.11), the proportion of fluid particles in a pore whose distance from the axis is between $r$ and $r+d r$ is $2 r d r / a^{2}$; hence for fluid particles

$$
\begin{aligned}
\int_{0}^{\infty} t f(t) d t & =\int_{0}^{a} \frac{2 r d r}{a^{2}} \int_{\Sigma} \frac{l}{2 \hat{q}\left(1-r^{2} / a^{2}\right)} \frac{\sin \theta}{2 \pi}\left(\frac{\lambda}{\pi}\right)^{\frac{3}{2}} \exp \left[-\lambda\left(p_{1}^{2}+p_{2}^{2}+p_{3}^{2}\right)\right] d \Sigma \\
& =\int_{0}^{1} \frac{d \rho}{1-\rho} \times \iint_{\substack{0<\theta<\pi \\
p+\cos \theta>0}} \frac{l}{6 U} \frac{\sin \theta}{p+\cos \theta}\left(\frac{\lambda}{\pi}\right)^{\frac{1}{2}} e^{-\lambda p^{2}} d p d \theta
\end{aligned}
$$

on substituting for $\hat{q}$ from (2.5) and (2.9), writing $\rho=r^{2} / a^{2}$, and using the method of integration described in the Appendix. Each of the integrals in (2.12) is divergent, and this as it turns out is the mathematical reason why it is necessary to make some attempt at estimating the effect of molecular diffusion on the motion through a pore.

Molecular diffusion affects the material quantity moving through a pore in two ways. First, the material quantity diffuses sideways across the pore so that an element of material quantity does not stay on a streamline $r=$ constant but spreads out over neighbouring streamlines. It may be expected that this effect is negligible if $t \ll t_{1}$, where $t$ is the time spent by the material element in the pore and $t_{1}$ is the time for appreciable molecular diffusion over a distance comparable with that in which the velocity differs by a factor of order unity. An estimate of the value of $t_{1}$ is $t_{1}=a^{2} / 8 \kappa$, where $\kappa$ is the molecular diffusivity (this expression is obtained by analogy from the formula for diffusion $x^{2}=2 \kappa t$ with $x=\frac{1}{2} a$ ).

If the average speed through the pore is so small that $l / \hat{q} \gg t_{1}$, then any material quantity has ample time to diffuse across the pore. This is the case discussed by Taylor $(1953,1954)$ and Aris (1956) who have shown that if a material quantity is introduced into a fluid flowing through a long thin straight tube, then after a time large compared with $t_{1}$ the concentration of the material quantity is approximately uniform over the pore cross-section and the material quantity is convected with the average speed in the tube, at the same time diffusing along the tube, relative to axes moving with the mean speed, with an effective diffusivity

$$
\kappa^{\prime}=\frac{a^{2} \hat{q}^{2}}{48 \kappa}+\kappa
$$


If we neglect for the moment the diffusion along the pore, it does not therefore seem unreasonable to suppose that the transport of material quantity along a pore for which $l / \hat{q} \gg t_{1}$ is approximately the same as the transport calculated on the assumption that elements of the material quantity remain elements and all move with the average speed. In other words, it is assumed that the duration of all steps through such a pore is $t=l / \hat{q}$.

If an element of material quantity enters a pore with $l / \hat{q} \ll t_{1}$ on a streamline close to the wall for which the duration of passage of an idealized fluid particle is large compared with $t_{1}$, then this element will have ample time to diffuse sideways on to streamlines where the velocity is not small and be convected along to the end of the pore. It again does not seem unreasonable to suppose that the transport of this material element is taken account of approximately by supposing that it remains an element and reaches the end of the pore in a time $t=t_{1}+l / \hat{q}$.

Let us now consider the second effect of molecular diffusion which is transport of material quantity by diffusion along the pore. This effect will be negligible if $t \ll t_{0}$, where $t_{0}=l^{2} / 2 \kappa$ is an estimate of the time for appreciable diffusion along the pore. If, on the other hand, $l / \hat{q} \gg t_{0}$, then the transport of a material element along the pore by convection will be negligible compared with the transport by diffusion, and it does not seem an unreasonable approximation to suppose that the transport along these pores is adequately represented by the passage of material elements in a time $t=t_{0}$. Note that the assumption $a \ll l$ implies $t_{1} \ll t_{0}$.

The preceding remarks may be combined in an approximate manner to give the following rule for specifying the duration of a step made by a material element:

$$
\begin{aligned}
t & =\frac{l}{2 \hat{q}\left(1-r^{2} / a^{2}\right)} \quad \text { if } \quad t \leqslant t_{1} \quad\left(t_{1}=a^{2} / 8 \kappa\right) \\
t & =t_{1}+l / \hat{q} \quad \text { if } \quad t_{1}<t \leqslant t_{0} \quad\left(t_{0}=l^{2} / 2 \kappa\right) \\
t & =t_{0} \quad \text { otherwise. }
\end{aligned}
$$

Now the above discussion of the effects of molecular diffusion is somewhat crude and limited, but it is not easy to see how it can be improved. The essential difficulty, which also occurs in the theory of turbulent diffusion, lies in the problem of combining the Lagrangian and Eulerian descriptions of a fluid motion, the former being the natural way of calculating how a marked particle moves and the latter of treating molecular diffusion. The rules (2.14) are probably alright if the molecular diffusivity is small enough for $t_{0}$ and $t_{1}$ to be large compared with the average duration $\bar{t}$, say, of a step (a precise value for $\bar{t}$ is obtained later but it is clearly $O(l / U)$ ), so that relatively few material elements are affected by molecular diffusion. Further, the values that will be obtained for the dispersion are logarithmic functions of $t_{0} / \vec{t}$ and $t_{1} / \bar{t}$, and are thus fairly insensitive to the numerical values in the definitions of $t_{0}$ and $t_{1}$. This reinforces belief in the adequacy of (2.14) to represent the effects of molecular diffusion. A smooth function of $\hat{q}, t_{0}, t_{1}$ and $r$ with essentially the same behaviour as (2.14), apart from the discontinuities at $t=t_{1}$ and $t=t_{0}$, may easily be constructed; but there is no real gain and the analysis becomes much more complicated.

It is possible that the structure of the medium is such that the pores should be regarded as exceptionally thin compared with their length and the average 
velocity is such that $t_{1} \ll \vec{t} \ll t_{0}$. In this case we shall suppose that the concentration of the material quantity is uniform over the pore cross-section and that the material quantity is convected with the average speed. The rule analogous to (2.14) for this case is

$$
\begin{aligned}
& t=l / \hat{q} \quad \text { if } t \leqslant t_{0} \quad\left(t_{0}=l^{2} / 2 \kappa\right), \\
& t=t_{0} \text { otherwise. }
\end{aligned}
$$

The objection may be raised that the effective diffusivity $\kappa^{\prime}$ as given by (2.13) should be used in the definition of $t_{0}$ rather than $\kappa$. However, when $t$ as given by $(2.14 b)$ or $(2.15 a)$ is close to $t_{0},\left(a^{2} \hat{Q}^{2} / 48 \kappa\right) / \kappa \doteqdot \frac{1}{12} a^{2} / l^{2}$, so that $\kappa^{\prime}$ may be replaced by $\kappa$ to a good approximation in the definition of $t_{0}$.

\section{The probability that a given step occurs in the random walk}

To complete the formulation of the random walk, it remains to evaluate the probability that a marked material element chooses a step with angles in the range $\theta$ to $\theta+d \theta$ and $\phi$ to $\phi+d \phi$, and of duration in the range $t$ to $t+d t$, where $t$ is defined as a function of $\hat{q}$ and $r$ by either (2.14) or (2.15), and $\hat{q}$ is a function of $\theta, \phi, p_{1}, p_{2}$ and $p_{3}$. The values of $\hat{q}$ and $r$ for a step by an infinitesimal material element are assumed to be the values of these quantities for the fluid particle with which it coincides at the beginning of the step (and remains coincident if $t_{1} \gg l /\left\{2 \hat{q}\left(1-r^{2} / a^{2}\right)\right\}$, otherwise they separate and the element completes the step in the time given by $(2.14)$ or $(2.15))$. The required probability is hence the probability that an idealized fluid particle chooses a streamline with values of $\theta, \phi, p_{1}, p_{2}, p_{3}$ and $r$ in the element of generalized volume $d \theta d \phi d p_{1} d p_{2} d p_{3} d r$. The proportion of pores with appropriate values of the variables is given by (2.7), and the proportion of a pore with $r$ in the range $r$ to $r+d r$ is $2 r d r / a^{2}$, and thus the proportion of such streamlines existing in the model is (2.7) multiplied by $2 r d r / a^{2}$, namely

$$
\frac{1}{2 \pi} \sin \theta\left(\frac{\lambda}{\pi}\right)^{\frac{3}{2}} \exp \left[-\lambda\left(p_{1}^{2}+p_{2}^{2}+p_{3}^{2}\right)\right] \frac{2 r}{a^{2}} d \theta d \phi d p_{1} d p_{2} d p_{3} d r
$$

Now this is not the probability that a fluid particle will choose such a streamline because the rate at which fluid particles go along a streamline is proportional to the velocity on the streamline. Suppose, for the sake of example, that a large number of particles arrive at a junction from which start two streamlines, then the relative number of fluid particles which go along the streamlines is proportional to the ratio of the velocities of the streamlines. Thus, the required probability is, apart from a normalizing factor, (2.16) multiplied by the velocity of the streamline, $2 \hat{q}\left(1-r^{2} / a^{2}\right)$, and is

$$
\frac{2}{N} \hat{q}\left(1-\frac{r^{2}}{a^{2}}\right) \frac{1}{2 \pi} \sin \theta\left(\frac{\lambda}{\pi}\right)^{\frac{3}{2}} \exp \left[-\lambda\left(p_{1}^{2}+p_{2}^{2}+p_{3}^{2}\right)\right] \frac{2 r}{a^{2}} d \theta d \phi d p_{1} d p_{2} d p_{3} d r
$$

where $\hat{q}$ is given by (2.5), the range of the variables is the domain $0 \leqslant \theta \leqslant \pi$, $0 \leqslant \phi<2 \pi, 0 \leqslant r \leqslant a, \hat{q} \geqslant 0$, denoted here by $P$, and $N$ is a normalizing factor so that the integral of (2.17) over $P$ is equal to unity. In fact, see the Appendix,

$$
N=\frac{3 U}{2}\left[\left(1+\frac{1}{2 \lambda}\right) \operatorname{erf} \sqrt{ } \lambda+\left(\frac{1}{\lambda \pi}\right)^{\frac{1}{2}} e^{-\lambda}\right] \text {. }
$$

For brevity of writing, we shall denote (2.17) symbolically by $d \mathbf{P}$. 


\section{Statistical independence of successive steps}

The remaining assumption to be made is that successive steps in the random walk are statistically independent. This is necessary in order to keep the analysis of the random walk tractable and seems alright as a beginning, but it is in fact not as good as might be thought. It appears reasonable as far as the values of $\theta$ and $r$ for successive steps are concerned since there is no apparent mechanism for correlating successive values, but this is not the case for $\hat{q}$ and $\phi$. Since fluid cannot accumulate, it follows that pores with large (or small) values of $\hat{q}$ are more likely to be followed by pores with large (or small) values of $\hat{q}$ than with small (or large) values. If many pores start and finish together, this effect is likely to be small; if only a few, then this effect will not be entirely negligible, but the indications are that the error due to this cause in the results to be obtained consists in the numerical coefficients being inaccurate by a factor not too different from unity.

The statistical independence of successive values of $\phi$ is rather more of a problem. However, it does not affect the longitudinal dispersion, owing to the statistical isotropy of the model, so we shall defer consideration until we examine the results for the lateral dispersion, which, of course, will depend upon whether there is correlation between successive values of $\phi$.

\section{Ensemble averages}

We can now evaluate the random variables $X_{n}, Y_{n}, Z_{n}$ and $T_{n}$, defined in (2.1) and (2.2), which give the displacement and time after $n$ steps of the random walk, and evaluate the probability that the displacement of an element of material quantity has a given value after a given time. This probability distribution is strictly speaking an ensemble average over many realizations of the model. That is, if we take a large number of samples of the medium, each with a different arrangement of pores but with the same statistical properties, and observe the displacement of a single material element through each of them, the calculated probability is equal to the proportion of samples in which the displacement has a given value after a given time as the number of samples tends to infinity. However, by the properties of ergodic theory, and assuming statistical homogeneity of the medium, the probability distribution gives the proportion of elements which have a given displacement after a given time if a large number of elements are started from different points scattered randomly through the same sample. All average properties of the random walk and of individual steps of the random walk are likewise ensemble averages, as distinct from spatial averages as in the definition of average velocity.

It is perhaps worth while demonstrating, for the sake of example, the equality of the spatial average and ensemble average (after many steps) of the velocity of an idealized fluid particle, i.e. a particle whose velocity is always given by (2.10). The (ensemble) average of the displacement after one step has components parallel to the axes:

$$
\begin{aligned}
& \bar{x}=\int_{P} l \cos \theta d \mathbf{P}=l U / N \quad \text { on performing the integration, } \\
& \bar{y}=\int_{P} l \sin \theta \cos \phi d \mathbf{P}=0, \quad \bar{z}=\int_{P} l \sin \theta \sin \theta d \mathbf{P}=0
\end{aligned}
$$


where $d \mathbf{P}$ is given by (2.17) and the bar denotes an ensemble average. The average duration of a step is

$$
\bar{t}=\int_{P} \frac{l}{2 \hat{q}\left(1-r^{2} / a^{2}\right)} d \mathbf{P}=l / N
$$

Then

$$
\bar{X}_{n}=\sum_{1}^{n} \bar{x}_{r}=n \bar{x}, \quad \bar{Y}_{n}=\bar{Z}_{n}=0, \quad \bar{T}_{n}=\sum_{1}^{n} \bar{t}_{r}=n \bar{t} .
$$

Hence, $\bar{X}_{n} / \bar{T}_{n}=U$ from which it follows that $X_{n} / T_{n} \rightarrow U$ in probability as $n \rightarrow \infty$, since (as follows rigorously from the results of the next section) $X_{n} / \bar{X}_{n}$ and $T_{n} \mid \bar{T}_{n}$ tend in probability to one as $n \rightarrow \infty$; and a fortior $i\left(\overline{X_{n} / T_{n}}\right) \rightarrow U$. Note that these results are independent of $\lambda$.

The dispersion of a cloud of marked particles can be calculated from the statistical properties of the displacement of a single marked particle if the time or displacement are large enough for the paths to become statistically independent.* Exactly how large the time or displacement should be for this condition to be approximately satisfied is far from clear, but it seems plausible that not too many, of the order of 100 say, will suffice for the longitudinal dispersion, although (for reasons which shall be given later) more may be required for the lateral dispersion.

$$
\text { The assumption } \lambda=\infty
$$

In the analysis of the random walk, we shall for the sake of mathematical simplicity put $\lambda=\infty$. This is equivalent to $\left(p_{1}, p_{2}, p_{3}\right)=0$. The average speed in a pore is then

$$
\hat{q}=3 U \cos \theta \quad\left(0 \leqslant \theta \leqslant \frac{1}{2} \pi\right),
$$

and the expression (2.17) for the probability of a step being such that $\theta, \phi$ and $r$ lie in a range of extent $d \theta d \phi d r$ becomes

$$
d \mathbf{P}=\frac{4}{\pi} \sin \theta \cos \theta \frac{r}{a^{2}}\left(1-\frac{r^{2}}{a^{2}}\right) d \theta d \phi d r,
$$

where the domain, $P$, of the variables is $0 \leqslant \theta \leqslant \frac{1}{2} \pi, 0 \leqslant \phi<2 \pi, 0 \leqslant r \leqslant a$. The rules (2.14) or (2.15) for the duration of a step by an element of material quantity remain unaltered.

There is no loss of physical generality in this assumption. The analysis may be carried out for a general value of $\lambda$, but it is found that the results for $\lambda$ finite differ from those for $\lambda=\infty$ only by a numerical factor of order $\operatorname{erf} \sqrt{ } \lambda(=1$ when $\lambda=\infty$ ). Now from (2.7), the proportion of steps, for a general value of $\lambda$, whose direction lies in the element of solid angle $\frac{1}{4} \pi \sin \theta d \theta d \phi$ is

$$
\frac{1}{2} \iiint_{Q>0}\left(\frac{\lambda}{\pi}\right)^{\frac{3}{2}} \exp \left[-\lambda\left(p_{1}^{2}+p_{2}^{2}+p_{3}^{2}\right)\right] d p_{1} d p_{2} d p_{3}=\frac{1}{2}\{1+\operatorname{erf}(\sqrt{ } \lambda \cos \theta)\}
$$

We may intuitively expect this number to be small when $\theta=\pi$, i.e. in the direction exactly opposite to that of the average velocity, so that there are reasonable

* I am indebted to Dr G. K. Batchelor for pointing out the proper interpretation of the mean values of the random walk as ensemble averages and the necessity for the paths of different marked particles to become statistically independent before the results for the displacement of a single particle may be used to calculate the dispersion of a cloud. 
grounds for believing that erf $\sqrt{ } \lambda$ is close to one. In view of the fact that the model is a considerable simplification of an actual porous medium, there is therefore no real loss in putting $\lambda=\infty$, especially as there is no point in making the analysis more complicated than is necessary and the analysis with $\lambda$ finite is lengthy and cumbersome.

\section{Analysis of the random walk}

This section is devoted to the calculation of the statistical properties of the displacement of a single marked element after many steps. It is mathematical in character and no further physical assumptions will be introduced. The results of the section will be summarized at the beginning of $\S 4$ for the benefit of the reader who does not wish to follow the mathematics in detail.

\subsection{Statistical properties of the displacement after many steps}

Consider the displacement of an element of the material quantity after $n$ steps, where $n$ is for the moment a fixed number. The components along the axes are given by (2.1). $X_{n}$ is the sum of $n$ independent random variables, and therefore

$$
\bar{X}_{n}=n l \overline{\cos \theta}
$$

similarly, $\bar{Y}_{n}=n l \overline{\sin \theta \cos \phi}$ and $\bar{Z}_{n}=n l \overline{\sin \theta \sin \phi}$, where the bar denotes an ensemble average and the probability distribution of $\theta$ and $\phi$ is given by (2.20). Thus, $\overline{\cos \theta}=\int_{P} \cos \theta d \mathbf{P}=\frac{2}{3}$ and $\overline{\sin \theta \cos \phi}=\overline{\sin \theta \sin \phi}=0$, the integrations being elementary. Hence,

$$
\bar{X}_{n}=\frac{2}{3} n l, \quad \bar{Y}_{n}=0, \quad \bar{Z}_{n}=0 .
$$

The variance of $X_{n}$ is the sum of the variances of the longitudinal displacement of the individual steps. Hence,

and similarly $\quad \bar{Y}_{n}^{2}=\bar{Z}_{n}^{2}=\frac{1}{4}\left(n l^{2}\right)=n l^{2} \sigma_{Y}^{2}$, say.

$$
\left.\begin{array}{rl}
\left(\bar{X}_{n}-\bar{X}_{n}\right)^{2} & =n l^{2} \int_{P}\left(\cos \theta-\frac{2}{3}\right)^{2} d \mathbf{P}=\frac{1}{18}\left(n l^{2}\right)=n l^{2} \sigma_{X}^{2}, \text { say } \\
\text { rly } \quad \bar{Y}_{n}^{2} & =\bar{Z}_{n}^{2}=\frac{1}{4}\left(n l^{2}\right)=n l^{2} \sigma_{Y}^{2}, \text { say. }
\end{array}\right\}
$$

Define dimensionless random variables

$$
\mathbf{x}_{n}=\frac{X_{n}-\bar{X}_{n}}{l n^{\frac{1}{2}}}=\frac{X_{n}}{l n^{\frac{1}{2}}}-\frac{2}{3} n^{\frac{1}{2}}, \quad \mathbf{y}_{n}=\frac{Y_{n}}{l n^{\frac{1}{2}}}, \quad \mathbf{z}_{n}=\frac{Z_{n}}{l n^{\frac{1}{2}}} .
$$

Then $\mathbf{x}_{n}, \mathbf{y}_{n}$ and $\mathbf{z}_{n}$ have zero mean and variances $\sigma_{X}^{2}, \sigma_{Y}^{2}, \sigma_{Y}^{2}$, respectively. Also, it is immediate that $\overline{\mathbf{x}_{n} \mathbf{y}_{n}}=\overline{\mathbf{x}_{n} \mathbf{z}_{n}}=\overline{\mathbf{y}_{n} \mathbf{z}_{n}}=0$. It follows from the Central Limit Theorem of statistics (see, for example, Cramér 1946) that the probability distributions of $\mathbf{x}_{n}, \mathbf{y}_{n}$ and $\mathbf{z}_{n}$ are asymptotically normal and statistically independent as $n \rightarrow \infty$, the departure from normality for a fixed value of $n$ being $O\left(n^{-\frac{1}{2}}\right)$.

\subsection{Statistical properties of the time for $n$ steps according to the rules (2.14)}

Consider now the time for $n$ steps, supposing that the rules (2.14) for the duration of a single step apply. (The rules (2.15) will be considered later.) These rules are an approximation and are expected to be valid only if $t_{1}$ and $t_{0}$ are large 
compared with the average duration of a step. We shall therefore neglect in the analysis all terms which vanish as $t_{0}$ and $t_{1}$ tend to infinity. The case of an idealized fluid particle is obtained by putting $t_{0}=t_{1}=\infty$. On substituting from (2.19) for $\hat{q}$ and using (2.14), we have for the average duration of a single step

$$
\bar{t}=\int_{P} t d \mathbf{P}=\int_{P_{1}} \frac{l}{6 U\left(1-r^{2} / a^{2}\right) \cos \theta} d \mathbf{P}+\int_{P_{\mathbf{2}}}\left(\frac{l}{3 U \cos \theta}+t_{1}\right) d \mathbf{P}+\int_{P_{3}} t_{0} d \mathbf{P},
$$

where the domain $P_{1}$ is that part of $P$ in which $l /\left\{6 U\left(1-r^{2} / a^{2}\right) \cos \theta\right\} \leqslant t_{1} ; P_{2}$ is that part of $P-P_{1}$ in which $t_{1}<t_{1}+l /(3 U \cos \theta)<t_{0} ;$ and $P_{3}=P-P_{1}-P_{2}$. Substituting for $d \mathbf{P}$ from (2.20), integrating with respect to $\phi$, and writing $\rho=1-r^{2} / a^{2}$, we obtain

$$
\bar{t}=\iint_{\mathrm{I}} \frac{2 l}{3 U} \sin \theta d \theta d \rho+\iint_{\mathrm{II}} 4\left(\frac{l}{3 U}+t_{1} \cos \theta\right) \rho \sin \theta d \theta d \rho+\iint_{\mathrm{III}} 4 t_{0} \rho \sin \theta \cos \theta d \theta d \rho,
$$

where the domain of integration $I$ is $0<\theta<\frac{1}{2} \pi, 0<\rho<1, \rho \cos \theta>l / 6 U t_{1}$; II is $0<\rho<1, \rho \cos \theta<l / 6 U t_{1}, \cos \theta>l /\left\{3 U\left(t_{0}-t_{1}\right)\right\} \doteqdot l / 3 U t_{0}$; and III is $0<\rho<1,0<\cos \theta<l / 3 U t_{0}$. The integration is straightforward and gives, neglecting terms which vanish as $t_{0}$ and $t_{1}$ tend to infinity,

$$
\bar{t}=2 l / 3 U,
$$

the largest term neglected being $-\left(l^{2} / 18 U^{2} t_{1}\right) \log \left(6 U t_{1} / l\right)$. It follows immediately that

$$
\bar{T}_{n}=2 n l / 3 U \text {. }
$$

The value of $(\overline{t-\bar{t}})^{2}=\int_{P}(t-\bar{t})^{2} d \mathbf{P}$ may be calculated in exactly the same manner. The calculation is straightforward, though a little lengthy, and gives

$$
\begin{aligned}
\overline{(t-\bar{t})^{2}} & =\frac{l^{2}}{U^{2}}\left\{\frac{2}{9} \log \frac{3 U t_{0}}{l}+\frac{1}{18}\left(\log \frac{6 U t_{1}}{l}\right)^{2}-\frac{1}{6} \log \frac{6 U t_{1}}{l}+\frac{1}{4}\right\} \\
& =\frac{l^{2}}{U^{2}} \sigma_{T}^{2}, \text { say. }
\end{aligned}
$$

Note that this variance does not exist if $t_{1}$ and $t_{0}$ are infinite, and we may therefore expect the statistical properties of the path of an idealized fluid particle to be very different from those of an element of material quantity, even though the molecular diffusivity is very small. This is why the rules (2.14) and (2.15) were formulated, a rough attempt at estimating the effects of molecular diffusion being thought better than none at all.

It follows from (3.6) that $\left(\overline{\left.T_{n}-\bar{T}_{n}\right)^{2}}=n l^{2} \sigma_{T}^{2} / U^{2}\right.$, and that the dimensionless random variable

$$
\mathbf{t}_{n}=\frac{T_{n}-\bar{T}_{n}}{(l / U) n^{\frac{1}{2}}}=\frac{T_{n}}{(l / U) n^{\frac{1}{2}}}-\frac{2}{3} n^{\frac{1}{2}}
$$

has zero mean and variance $\sigma_{T}^{2}$.

Since $\sigma_{T}$ is large when $t_{1}$ and $t_{0}$ are large compared with $l / U$, we must be cautious in applying the Central Limit Theorem to $t_{n}$. As a rough estimate (a more precise 
argument based on the results of $\$ 3.7$ below gives a slightly different one), $t_{n}$ will be normally distributed when $\mathbf{t}_{n}^{3}$ is small compared with one. Now,

$$
\overline{\left(T_{n}-\bar{T}_{n}\right)^{3}}=\overline{n(t-\bar{t})^{3}}
$$

and it may be shown that $\overline{(t-\bar{t})^{3}} \sim \frac{1}{3} l^{2} t_{0} / U^{2}$; thus

$$
\mathbf{\mathbf { t }}_{n}^{3} \sim \frac{1}{3} \frac{U t_{0}}{\ln \frac{1}{2}} .
$$

Hence, $\mathbf{t}_{n}$ is normally distributed to a good approximation only if the number of steps is so large that $n^{\frac{1}{2}} \gg \frac{1}{3} U t_{0} / l$; i.e. the smaller $\kappa$ and the larger $t_{0}$, then the larger is the number of steps before (3.7) is normally distributed.

The covariance of $\mathbf{x}_{n}$ and $\mathbf{t}_{n}$ is

$$
\overline{\mathbf{x}_{n} \mathbf{t}_{n}}=\frac{\overline{\left(\overline{\left.X_{n}-\bar{X}_{n}\right)\left(T_{n}-\bar{T}_{n}\right.}\right)}}{n l^{2} / U}=\frac{\overline{(\cos \theta-\overline{\cos \theta})(t-\bar{t}})}{l / U}=-\frac{1}{9}=\sigma_{X T}, \text { say, }
$$

terms which vanish when $t_{1}$ and $t_{0}$ equal infinity being neglected. It is immediate that $\overline{\mathbf{y}_{n} \mathbf{t}_{n}}=\overline{\mathbf{z}_{n} \mathbf{t}_{n}}=0$, because the terms in $\phi$ integrate to zero.

\subsection{Probability distribution of the number of steps in a given time}

So far we have examined the displacement and time after $n$ steps, where $n$ is a fixed number. In order to calculate the dispersion, it is necessary to know the probability distribution (p.d.) of the displacement after a given time $T$, say, where $T$ is in general large compared with $l / U$.

The first step is to calculate the p.d. of the number of steps made in the time $T$. The probability that more than $n$ steps are required before the time exceeds $T$ is equal to the probability that $T_{n}<T$, since each event implies the other, which in turn is equal to the probability that $\mathbf{t}_{n}<\mathbf{t}$, where

$$
\mathbf{t}=\frac{T}{(l / U) n^{\frac{1}{2}}}-\frac{2}{3} n^{\frac{1}{2}}
$$

Hence, the probability that $\mathbf{t}$ (with $T$ fixed and $n$ variable) lies in the range $\mathbf{t}$ to $\mathbf{t}+d \mathbf{t}$ is equal to the probability that $\mathbf{t}_{n}$ lies between $\mathbf{t}$ and $\mathbf{t}+d \mathbf{t}$ when the value of $n$ satisfies (3.10), i.e.

$$
n=\frac{3}{2} \frac{U T}{l}+\frac{9}{8} \mathbf{t}^{2}-\frac{9 \mathbf{t}}{8}\left(\mathbf{t}^{2}+\frac{8 U T}{3 l}\right)^{\frac{1}{2}} .
$$

(The positive square root is taken in (3.11) so that $n$ goes from 0 to $\infty$ as $t$ goes from $+\infty$ to $-\infty$. Also, it is supposed that $n$ is sufficiently large for it to be regarded as a continuous variable.)

The p.d. of $\mathbf{t}$ is in general a complicated function, being dependent upon the p.d. of $\mathbf{t}_{n}$ for all $n$, but if the mean value of $n$ for given $T$, denoted by $\bar{n}$, is large, the p.d. of $t$ will depend mainly upon the p.d.'s of $t_{n}$ for values of $n$ not too different from $\bar{n}$ and the p.d. of $t$ will approximate to that of $t_{\bar{n}}$. Indeed, as $\bar{n} \rightarrow \infty$ the p.d.'s of $t$ and $t_{\bar{n}}$ become identical since the p.d. of $t_{n}$ is independent of $n$ for sufficiently large $n$. 
Equation (3.11) may be written approximately as

$$
n=\frac{3}{2} \frac{U T}{l}-\frac{9 \mathbf{t}}{8}\left(\frac{8 U T}{3 l}\right)^{\frac{1}{2}}
$$

the terms neglected being $O\left(\sigma_{T}^{2}\right)$. It follows that

with error $O\left(\sigma_{T}^{2} / \bar{n}\right)$.

$$
\bar{n}=\frac{3}{2} \frac{U T}{l}
$$

\subsection{The p.d. of the lateral displacement after a given time}

Denote the component of the displacement in the $y$-direction after time $T$ by $Y$. From (3.3), $Y=\ln ^{\frac{1}{2}} \mathbf{y}_{n}$, and if $\bar{n}^{\frac{1}{2}} \gg 1$ we may write $\mathbf{y}_{n}=\mathbf{y}_{\bar{n}}$ except for a negligible set of values of $n$. Further, if $\left.\sigma_{T}\right|^{\frac{1}{2}} \ll 1, n$ is given by (3.12) and, to the same approximation,

$$
n^{\frac{1}{2}}=\left(\frac{3}{2} \frac{U T}{l}\right)^{\frac{1}{2}}-\frac{3}{4} \mathbf{t}
$$

Hence

$$
Y=\left(\frac{3}{2} U T l\right)^{\frac{1}{2}} \mathbf{y}_{\bar{n}}-\frac{3}{4} \mathbf{t y} \mathbf{y}_{\bar{n}} \doteqdot\left(\frac{3}{2} U T l\right)^{\frac{1}{2}} \mathbf{y}_{\bar{n}} \text {. }
$$

Thus, when $\sigma_{T} / \bar{n}^{\frac{1}{2}} \ll 1, Y$ is normally distributed with zero mean and

on substituting from (3.2).*

$$
\bar{Y}^{2}=\frac{3}{2} U l T \sigma_{Y^{r}}^{2}=\frac{3}{8} U l T,
$$

Identical results hold for the displacement in the $z$-direction. Note that the p.d. of the lateral displacement is independent of molecular diffusion and the same result should hold for idealized fluid particles; it will be verified later that this is indeed so.

\subsection{The p.d. of the longitudinal displacement after a given time}

Denote the longitudinal displacement after time $T$ by $X$. From (3.3)

$$
X=\ln ^{\frac{1}{2}} \mathbf{x}_{n}+\frac{2}{3} \ln ,
$$

and if $\bar{n}^{\frac{1}{2}} \gg 1$, we may again write $\mathbf{x}_{n}=\mathbf{x}_{\bar{n}}$. Substituting for $n$ from (3.12) and (3.14), we have

$$
X=U T-\left(\frac{3}{2} U T l\right)^{\frac{1}{2}}\left(\mathbf{t}-\mathbf{x}_{\bar{n}}\right)
$$

neglecting terms of order $l \mathbf{t} \mathbf{x}_{\bar{n}}$. Then

$$
\vec{X}=U T=\frac{2}{3} l \bar{n} .
$$

Suppose now that $T$ is sufficiently large for

$$
\bar{n}^{\frac{1}{2}} \gg \frac{1}{3} \frac{U t_{0}}{l} .
$$

Then the p.d. of $t_{\bar{n}}$ is normally distributed with variance $\sigma_{T}^{2}$, and so also will be the p.d. of $t$. Hence,

$$
\frac{X-U T}{T^{\frac{1}{2}}}=-\left(\frac{3}{2} U l\right)^{\frac{1}{2}}\left(\mathbf{t}-\mathbf{x}_{\bar{n}}\right)
$$

* In the interests of conciseness, the argument is presented here and in $\$ 3.5$ in an heuristic manner. Rigorous arguments can be formulated, but they are more complicated and less direct, and appear to be out of place in the present context. 
is the sum of two normally distributed variables with finite variances and is therefore itself normally distributed with zero mean and variance given by

$$
\begin{aligned}
\frac{1}{T} \overline{(X-U T)^{2}} & =\frac{3}{2} U l\left(\overline{\mathbf{t}^{2}}-2 \overline{\mathbf{t} \mathbf{x}_{\bar{n}}}+\overline{\mathbf{x}_{\bar{n}}^{2}}\right)=\frac{3}{2} U l\left(\sigma_{T}^{2}+\sigma_{X}^{2}-2 \sigma_{X T}\right) \\
& =U l\left\{\frac{1}{3} \log \frac{3 U t_{0}}{l}+\frac{1}{12}\left(\log \frac{6 U t_{1}}{l}\right)^{2}-\frac{1}{4} \log \frac{6 U t_{1}}{l}+\frac{19}{24}\right\}
\end{aligned}
$$

on substituting from (3.2), (3.6) and (3.9).

It should perhaps be mentioned that this analysis proves only that the p.d. of $X / T^{\frac{1}{2}}$ is asymptotically normal, it does not prove that the p.d. of $X-U T$ is asymptotically normal. Indeed, from (3.16) and (3.8),

$$
(X-U T)^{3} \sim-\left(\frac{3}{2} U T l\right)^{\frac{3}{2}} \overline{\mathbf{t}}^{3} \sim-\frac{3}{4} U^{2} T l t_{0},
$$

which becomes large as $T \rightarrow \infty$, so that the analysis predicts that the p.d. of $X$, as opposed to that of $X / T^{\frac{1}{2}}$, becomes increasingly skew as $T$ increases.

\subsection{The random walk according to the rules (2.15)}

The results obtained so far have been based on the rules (2.14) which are for the case $l / U \ll t_{1} \ll t_{0}$. It is expected, however, that the rules (2.15) for the case $t_{1} \ll l U \ll t_{0}$ may sometimes apply.

The previous analysis depends upon the duration of a single step only in so far as the statistical properties of $T_{n}$ are affected. That is to say, the previous analysis is unaffected apart from $\$ 3.2$ which must be repeated using the new rules. It is then sufficient to replace the value of $\sigma_{T}$ used above by a new value, $\sigma_{T_{1}}$ say, obtained using (2.15).

We have

$$
\bar{t}=\int_{P} t d \mathbf{P}=\int_{P_{1}} \frac{l}{3 U \cos \theta} d \mathbf{P}+\int_{P_{2}} t_{0} d \mathbf{P},
$$

where $P_{1}$ is that part of $P$ in which $l /\{3 U \cos \theta\}<t_{0}$, and $P_{2}=P-P_{1}$. Substituting for $d \mathbf{P}$ from (2.20), integrating with respect to $\phi$ and $r$, and neglecting terms which vanish as $t_{0} \rightarrow \infty$ (these are at most $l^{2} / 9 U^{2} t_{0}$ ), one obtains

$$
\bar{t}=\int_{\cos \theta=l / 3 U t_{0}}^{1} \frac{2 l}{3 U} \sin \theta d \theta+\int_{\cos \theta=0}^{l / 3 U t_{0}} 2 t_{0} \sin \theta \cos \theta d \theta=\frac{2 l}{3 U}
$$

as before. Similarly, the variance of the duration may be calculated, giving

$$
\overline{(t-\bar{t})^{2}}=\frac{l^{2}}{U^{2}}\left\{\frac{2}{9} \log \frac{3 U t_{0}}{l}-\frac{1}{3}\right\}=\frac{l^{2}}{\bar{U}^{2}} \sigma_{T_{1}}^{2}, \quad \text { say. }
$$

The values of $\overline{\mathbf{x}_{n} \mathbf{t}_{n}}$ and $\overline{\mathbf{t}_{n}^{3}}$ are again given by (3.9) and (3.8).

The results of $\S \S 3.4$ and 3.5 now apply with $\sigma_{T}$ replaced by $\sigma_{T_{1}}$. Thus, the statistical properties of the lateral displacement remain unaltered. So do those of $X$ except that now

$$
\frac{1}{T} \overline{(X-U T)^{2}}=U l\left\{\frac{1}{3} \log \frac{3 U t_{0}}{l}-\frac{1}{12}\right\}
$$

when (3.18) is satisfied.

It seems plausible to suppose that the lateral displacement is independent of $t_{1}$, since the same result is obtained for $t_{1} \gg l / U$ and $t_{1} \ll l / U$. For the longitudinal 
displacement, the leading terms of (3.19) and (3.22) are the same and it seems plausible that the variance of the displacement for intermediate values of $t_{1}$ will be intermediate between the values of (3.19) and (3.22).

\subsection{Further analysis of the random walk}

The reason why the statistical properties of the longitudinal displacement involve the molecular diffusivity (through $t_{1}$ and $t_{0}$ ) is that the mean square duration of a step by an idealized fluid particle is not finite, ${ }^{*}$ and therefore the Central Limit Theorem cannot be applied to the variable $t_{n}$ evaluated for such particles. It is then not possible to prove that $(\mathrm{I} / T)\left(\overline{X-U T)^{2}}\right.$ tends asymptotically to a value independent of $T$ as is usually the case for random processes occurring in nature. As we have seen, this difficulty does not arise if molecular diffusion is taken into account. However, the expressions (3.19) and (3.22) tend to infinity as $\kappa \rightarrow 0$, and also the value of $T$ which must obtain for these expressions to apply becomes infinite as $\kappa \rightarrow 0$, because of (3.18).

It is natural to inquire what happens if $\kappa$, or more precisely $l / U t_{0}$, is so small that (3.18) is not satisfied even though $T$ is sufficiently large for $\bar{n}^{\frac{1}{2}} \gg 1$, or alternatively what are the statistical properties of the displacement of an idealized fluid particle whose velocity is given by (2.10) for all values of $t$. To answer these questions, it is necessary to investigate the statistical properties of the random walk in rather more detail.

Consider the joint probability distribution function (j.p.d.f.) of $\mathbf{x}_{n}, \mathbf{y}_{n}, \mathbf{z}_{n}$ and $t_{n}$, where these random variables are defined by (3.3) and (3.7) but for the moment we do not specify the value of the duration of a step. The joint characteristic function (j.c.f.) of these quantities is the fourier transform of the j.p.d.f.; that is, the j.c.f., $C_{n}(\xi, \eta, \zeta, \tau)$ say, is the expectation of $\exp i\left(\xi \mathbf{x}_{n}+\eta \mathbf{y}_{n}+\zeta \mathbf{z}_{n}+\tau \mathbf{t}_{n}\right)$. By well-known results (see Cramér 1946), if $\Phi(\xi, \eta)$ is the j.c.f. of random variables $x$ and $y$ with a j.p.d.f. $\phi(x, y)$, the j.c.f. of $a x+b$ and $c y+d(a, b, c$ and $d$ being constants) is $e^{b \xi+d \eta} \Phi(a \xi, c \eta)$. Also, the j.c.f. of $\sum_{1}^{n} x_{r}$ and $\sum_{1}^{n} y_{r}$ is $\{\Phi(\xi, \eta)\}^{n}$.
It follows from these results that

$$
\begin{aligned}
& C_{n}(\xi, \eta, \zeta, \tau) \\
& \quad=\left\{\int_{P} d \mathbf{P} \exp \frac{i}{n^{\frac{1}{2}}}\left[\xi(\cos \theta-\overline{\cos \theta})+\eta \sin \theta \cos \phi+\zeta \sin \theta \sin \phi+\frac{U \tau}{l}(t-\bar{t})\right]\right\}^{n}
\end{aligned}
$$

We require the value of (3.23) when $n$ is large compared with unity. The analysis is not particularly difficult, but the details are very tedious and we shall confine ourselves to a brief description of the principal steps. The essential difficulty is that the exponential in the integrand in (3.23) may be expanded as a power series in $\xi, \eta$ and $\zeta$, but not in $\tau$, since $\tau$ is multiplied by $t$ which ranges up to $t_{0}$ (or infinity), unless $U t_{0} / l n^{\frac{1}{2}} \ll 1$ which is now not necessarily the case.

* The mean square duration of a step by an idealized fluid particle is $\int t^{2} d \mathbf{P}$ which is proportional to $\int_{0}^{\infty} t f(t) d t$, where $f(t) d t$ is the (spatial) proportion of particles at any instant undergoing steps of duration between $t$ and $t+d t$, since, as explained in $\S 2, d \mathbf{P}$ is proportional to this proportion multiplied by the velocity of the step. According to (2.12), this integral diverges for all $\lambda$ so that the above result does not arise from the assumption $\lambda=\infty$. 
Suppose now that $d \mathbf{P}$ is given by (2.20), and $t$ is given by one of $(2.11),(2.14)$ or (2.15). We then take the logarithm of (3.23) and expand the exponential as a power series in $\xi, \eta$ and $\zeta$, giving after some reduction

$$
\begin{aligned}
\log C_{n}=n\left\{\int_{P} \exp \left[i U \tau(t-\bar{t}) / l n^{\frac{1}{2}}\right] d \mathbf{P}-1\right\} \\
-\frac{1}{2}\left(\xi^{2} \sigma_{X}^{2}+\eta^{2} \sigma_{Y}^{2}+\zeta^{2} \sigma_{Y}^{2}+2 \xi \tau \sigma_{X T}\right)+O\left(n^{-\frac{1}{2}}\right),
\end{aligned}
$$

where use has been made of the result

$$
\int_{P} f(\theta, \phi) \exp \left[i U \tau t / \ln n^{\frac{1}{2}}\right] d \mathbf{P}=\int_{P} f(\theta, \phi)\left\{1+i \frac{U \tau t}{l n^{\frac{1}{2}}}\right\} d \mathbf{P}+o\left(n^{-\frac{1}{2}}\right)
$$

for $f(\theta, \phi)$ a regular function, and the values of $\sigma_{X}^{2}, \sigma_{Y}^{2}$ and $\sigma_{X T}$ are those given in (3.2) and (3.9). The result (3.25) follows from the fact that differentiation with respect to $\tau$ under the integral sign is permissible if the resulting integral is absolutely convergent.

It remains to evaluate

$$
n\left\{\int_{P} \exp \left[i U \tau \frac{(t-\bar{t})}{\ln \frac{1}{2}}\right] d \mathbf{P}-1\right\}=-\frac{1}{2} \Sigma^{2} \tau^{2}, \text { say. }
$$

Suppose the rules (2.14) apply. Then the integral may be broken up as in the derivation of (3.4) into integrals with respect to $\theta$ and $\rho$. In the domain I (see $\S 3.3)$ introduce new variables $\psi=\tau /\left(6 n^{\frac{1}{2}} \rho \cos \theta\right), \rho=\rho$; and in the domain II put $\psi=\tau /\left(3 n^{\frac{1}{2}} \cos \theta\right)$. Integration with respect to $\rho$ gives after some reduction

$$
\begin{aligned}
& \Sigma^{2}=-\frac{2}{9} \exp \left[-i U \tau \bar{t} / l n^{\frac{1}{2}}\right] \int_{\tau / 6 n^{\frac{1}{3}}}^{U t_{1} \tau / l n^{i}} \frac{e^{i \psi}}{\psi^{3}} \log \frac{6 n^{\frac{1}{2}} \psi}{\tau} d \psi \\
& -\frac{2 n l^{2}}{9 U^{2} t_{0}^{2} \tau^{2}} \exp \left[i U \tau\left(t_{0}-\bar{t}\right) / l n^{\frac{1}{2}}\right]+\frac{2 n}{\tau^{2}}-\exp \left[i U \tau\left(t_{1}-\bar{t}\right) / l n^{\frac{1}{2}}\right] \\
& \times\left\{\frac{4}{9} \int_{2 U t_{1} \tau / l n^{\natural}}^{U t_{0} \tau / l n^{\ddagger}} e^{i \psi} d \psi / \psi^{3}+\frac{n l^{2}}{9 U^{2} t_{1}^{2} \tau^{2}} \int_{\tau / 3 n^{\ddagger}}^{2 U t_{1} \tau / l n^{\ddagger}} \frac{e^{i \psi}}{\psi} d \psi\right\} .
\end{aligned}
$$

By integration by parts and use of the results

$$
\begin{gathered}
\int_{1 / \epsilon}^{\infty} \frac{e^{i \psi}}{\psi} d \psi=O(\epsilon), \quad \int_{1 / \epsilon}^{\infty} \frac{e^{i \psi}}{\psi} \log \psi d \psi=O(\epsilon \log (1 / \epsilon)), \\
\int_{\epsilon}^{\infty} \frac{e^{i \psi}}{\psi} d \psi=-\log \epsilon+\left(\gamma+i\left(\frac{1}{2} \pi\right)\right)+O(\epsilon), \\
\int_{\epsilon}^{\infty} \frac{e^{i \psi}}{\psi} \log (\psi / \epsilon) d \psi=\frac{1}{2}(\log \epsilon)^{2}+\left(\gamma+i\left(\frac{1}{2} \pi\right)\right) \log \epsilon+O(1),
\end{gathered}
$$

where $\gamma$ is Euler's constant (see Jeffreys \& Jeffreys 1950, p. 471), it may be shown that $\Sigma^{2}$ is given approximately by

$$
\begin{aligned}
& \Sigma^{2}= \frac{2}{9} \log \frac{3 U t_{0}}{l}+\frac{1}{18}\left(\log \frac{6 U t_{1}}{l}\right)^{2}-\frac{1}{6} \log \frac{6 U t_{1}}{l}+\frac{1}{4} \quad \text { if } \quad \frac{\tau U t_{0}}{3 \ln \frac{1}{2}} \ll 1 ; \\
& \Sigma^{2}=\frac{2}{9} \log \frac{3 n^{\frac{1}{2}}}{\tau}+\frac{1}{18}\left(\log \frac{6 U t_{1}}{l}\right)^{2}-\frac{1}{6} \log \frac{6 U t_{1}}{l}+\frac{1}{4} \\
& \quad \text { if } \log \frac{3 n^{\frac{1}{2}}}{\tau} \gg 1, \frac{\tau U t_{1}}{l n^{\frac{1}{2}}} \ll 1, \quad \frac{\tau U t_{0}}{l n^{\frac{1}{2}}} \gg 1 ; \\
& \Sigma^{2}=\frac{1}{18}\left(\log \frac{6 n^{\frac{1}{2}}}{\tau}\right)^{2} \quad \text { if } \log \frac{6 n^{\frac{1}{2}}}{\tau} \gg 1, \frac{\tau U t_{1}}{\ln \frac{1}{2}} \gg 1, \frac{\tau U t_{0}}{\ln \frac{1}{2}} \gg 1 .
\end{aligned}
$$


Actually, the conditions given here for these formulae to hold are more restrictive than is strictly necessary, but more precise conditions are rather complicated.

Similarly, if the rules (2.15) hold,

$$
\begin{aligned}
& \Sigma^{2}=\frac{2}{9} \log \frac{3 U t_{0}}{l}-\frac{1}{3} \quad \text { if } \quad \frac{\tau U t_{0}}{3 \ln \frac{1}{2}} \ll 1 \\
& \Sigma^{2}=\frac{2}{9} \log \frac{3 n^{\frac{1}{2}}}{\tau} \quad \text { if } \quad \log \frac{3 n^{\frac{1}{2}}}{\tau} \gg 1, \quad \frac{\tau U t_{0}}{\ln \frac{1}{2}} \gg 1 .
\end{aligned}
$$

The j.p.d.f. of $\mathbf{x}_{n}, \mathbf{y}_{n}, \mathbf{z}_{n}$ and $\mathbf{t}_{n}$ is the fourier transform of $C_{n}$ and is, for $n^{\frac{1}{2}} \gg 1$,

$$
\begin{aligned}
\left(\frac{1}{2 \pi}\right)^{2} \iiint \int \exp \left[i\left(\xi \mathbf{x}_{n}+\eta \mathbf{y}_{n}+\zeta \mathbf{z}_{n}+\tau \mathbf{t}_{n}\right)\right] \\
\quad \times \exp \left[-\frac{1}{2}\left(\xi^{2} \sigma_{X}^{2}+\eta^{2} \sigma_{Y}^{2}+\zeta^{2} \sigma_{Y}^{2}+\tau^{2} \Sigma^{2}+2 \xi \tau \sigma_{X T}\right)\right] d \xi d \eta d \zeta d \tau .
\end{aligned}
$$

If $\Sigma$ were constant, it would follow immediately that the j.p.d.f. is normal with $\overline{\mathbf{x}_{n}^{2}}=\sigma_{X}^{2}, \overline{\mathbf{y}_{n}^{2}}=\overline{\mathbf{z}_{n}^{2}}=\sigma_{Y}^{2}, \overline{\mathbf{t}_{n}^{2}}=\Sigma^{2}$ and $\overline{\mathbf{x}_{n} \mathbf{t}_{n}}=\sigma_{X T}$, the other covariances being zero. Now $\Sigma$ is actually a function of $\tau$, but it is a slowly varying function and the main contribution to the integration with respect to $\tau$ comes from values of $\tau$ for which $\Sigma \tau=O(1)$, and it may be proved that to a sufficient approximation (3.29) may be evaluated with $\Sigma$ independent of $\tau$ and having the value determined by $\Sigma \tau=1$.

It follows that for the rules (2.14), the value of $\Sigma^{2}\left(=\overline{\mathbf{t}_{n}^{2}}\right)$ is approximately

$$
\begin{gathered}
\Sigma^{2}=\frac{2}{9} \log \frac{3 U t_{0}}{l}+\frac{1}{18}\left(\log \frac{6 U t_{1}}{l}\right)^{2}-\frac{1}{6} \log \frac{6 U t_{1}}{l}+\frac{1}{4} \quad \text { if } \frac{U t_{0}}{n^{\frac{1}{2}} l\left(\log 3 U t_{0} / l\right)^{\frac{1}{2}}} \ll 1 ; \\
\Sigma^{2}=\frac{2}{9} \log 3 n^{\frac{1}{2}}+\frac{1}{18}\left(\log \frac{6 U t_{1}}{l}\right)^{2}-\frac{1}{6} \log \frac{6 U t_{1}}{l}+\frac{1}{4} \text { if } \log n^{\frac{1}{2}} \gg 1, \\
\frac{3 U t_{1}}{n^{\frac{1}{2}} l\left(\log n^{\frac{1}{2}}\right)^{\frac{1}{2}}} \ll 1, \quad \frac{3 U t_{0}}{n^{\frac{1}{2} l}\left(\log n^{\frac{1}{2}}\right)^{\frac{1}{2}}} \gg 1 ;
\end{gathered}
$$

and for the rules (2.15)

$$
\begin{gathered}
\Sigma^{2}=\frac{2}{9} \log \frac{3 U t_{0}}{l}-\frac{1}{4} \quad \text { if } \quad \frac{U t_{0}}{n^{\frac{1}{2}} l\left(\log 3 U t_{0} l\right)^{\frac{1}{2}}} \ll 1 ; \\
\Sigma^{2}=\frac{2}{9} \log 3 n^{\frac{1}{2}} \quad \text { if } \quad \log n^{\frac{1}{2}} \gg 1, \quad \frac{3 U t_{0}}{n^{\frac{1}{2}} l\left(\log n^{\frac{1}{2}}\right)^{\frac{1}{2}}} \gg 1 .
\end{gathered}
$$

The cases $(3.30 a)$ and $(3.31 a)$ are those which we have in fact already considered when $(3.18)$ is satisfied. The case $(3.30 \mathrm{c})$ describes the motion of an idealized fluid particle since these conditions apply when $t_{1}$ and $t_{0}$ become infinite.

3.8. The statistical properties of the displacement after a fixed time

The analysis of $\S 3.3$ for the probability distribution of the number of steps in a given time may now be repeated with the variance of $t_{n}$ given by the above results. The statistical properties of $t$ will again not be very different from those of $t_{\bar{n}}$, the difference between them becoming smaller as $\bar{n}$ becomes larger since $\Sigma$ is a slowly varying function of $n$. 
The analysis of $\$ 3.4$ and 3.5 for the lateral and longitudinal displacement may then be repeated with $\sigma_{T}$ replaced by $(\Sigma)_{n=\bar{n}}$. Thus $Y$ is normally distributed in all cases with zero mean and variance given by (3.15), and $(X-U T) / T^{\frac{1}{2}}$ is normally distributed with zero mean and variance

$$
\frac{1}{T} \overline{(X-U T)^{2}}=\frac{3}{2} U l\left\{\left(\Sigma^{2}\right)_{n=\bar{n}}+\sigma_{X}^{2}-2 \sigma_{X T}\right\}=U l S^{2}, \quad \text { say. }
$$

It seems worth adding here a word of comment about the apparent contradiction between the results of $(3.30 b),(3.30 c)$ and $(3.31 b)$ and the fact that $t_{n}$ has exactly the variance $\sigma_{T}^{2}$ or $\sigma_{T_{1}}^{2}$, as the case may be, for all $n$. The explanation is that the p.d. of $t_{n}$ is approximately that of a normal distribution with variance $\Sigma^{2}$ provided the value of $t_{n}$ is not too large. For very large values of $t_{n}$, the p.d. is significantly different from that of the normal distribution with variance $\Sigma^{2}$, and it is this tail which makes the exact p.d. of $\mathbf{t}_{n}$ have variance $\sigma_{T}^{2}$ or $\sigma_{T_{2}}^{2}$. Examination of the analysis of $\$ 3.3$ shows that only the values of $t$ in the neighbourhood of $\mathbf{t}=0$ are relevant to the calculation of $X-U T$, and that the long tail to the p.d. of $\mathbf{t}_{n}$ may be neglected.

\section{The lateral and longitudinal dispersion}

We obtain from the analysis of the previous section the following results for the statistical properties of the displacement of a single particle after a given (large) time $T$.

The mean number of steps and mean longitudinal displacement are, respectively,

$$
\bar{n}=\frac{3}{2} \frac{U T}{l} \text { and } \quad \bar{X}=U T \quad \text { if } \quad \bar{n} \gg 1 .
$$

The mean lateral displacement is $\bar{Y}=\bar{Z}=0$.

The lateral displacement is normally distributed and

$$
\bar{Y}^{2}=\bar{Z}^{2}=\frac{3}{8} U l T \text { if } \quad \bar{n}^{\frac{1}{2}} \gg 1 \text {. }
$$

For the longitudinal displacement when the rules (2.14) apply, necessary conditions being $U t_{1} / l \gg 1, U t_{0} / l \gg 1$ where $t_{1}=a^{2} / 8 \kappa, t_{0}=l^{2} / 2 \kappa$, then $(X-U T) / T^{\frac{1}{2}}$ is approximately normally distributed with variance $U l S^{2}$ given by (3.32): from (3.30) the approximate values are given by

$$
\begin{aligned}
& S^{2}=\frac{1}{3} \log \frac{3 U t_{0}}{l}+\frac{1}{12}\left(\log \frac{6 U t_{1}}{l}\right)^{2}-\frac{1}{4} \log \frac{6 U t_{1}}{l}+\frac{19}{2} \frac{\text { if }}{\bar{n}^{\frac{1}{2}}\left(\log 3 U t_{0} / l\right)^{\frac{1}{2}}} \ll 1 ; \\
& S^{2}=\frac{1}{6} \log \frac{27 U T}{2 l}+\frac{1}{12}\left(\log \frac{6 U t_{1}}{l}\right)^{2}-\frac{1}{4} \log \frac{6 U t_{1}}{l}+\frac{19}{24} \\
& \text { if } \log \bar{n}^{\frac{1}{2}} \gg 1, \quad \frac{3 U t_{1} l}{\bar{n}^{\frac{1}{2}}\left(\log \bar{n}^{\frac{1}{2}}\right)^{\frac{1}{2}}} \ll 1, \quad \frac{3 U t_{0} / l}{\bar{n}^{\frac{1}{2}}\left(\log \bar{n}^{\frac{1}{2}}\right)^{\frac{1}{2}}} \gg 1 ; \\
& S^{2}=\frac{1}{48}\left(\log \frac{54 U T}{l}\right)^{2} \quad \text { if } \log \bar{n}^{\frac{1}{2}} \gg 1, \quad \frac{4 U t_{1} / l}{\bar{n}^{\frac{1}{2}} \log \bar{n}^{\frac{1}{2}}} \gg 1, \quad \frac{4 U t_{0} / l}{\bar{n}^{\frac{1}{2}} \log \bar{n}^{\frac{1}{2}}} \gg 1
\end{aligned}
$$

* This result is also obtained by Josselin de Jong (1958). 
Thus idealized fluid particles $\left(t_{0}=t_{1}=\infty\right)$ obey $(4.3 \mathrm{c})$, and the variance of their longitudinal displacement is an increasing function of $T$. This is also the case for elements of a material quantity subject to molecular diffusion provided the time for appreciable molecular diffusion across streamlines is sufficiently large compared with $T$; but as $T$ (and $\bar{n}$ ) increase, the variance is given by $(4.3 b)$ and eventually by $(4.3 a)$. The value of $S^{2}$ is then independent of $T$.

If the rules (2.15) apply, necessary conditions being $U t_{1} / l \ll 1$ and $U t_{0} / l \gg 1$, then from (3.31) approximate values of $S^{2}$ are

$$
\begin{aligned}
S^{2} & =\frac{1}{3} \log \frac{3 U t_{0}}{l}-\frac{1}{12} \quad \text { if } \quad \frac{U t_{0} / l}{\bar{n}^{\frac{1}{2}}\left(\log 3 U t_{0} / l\right)^{\frac{1}{2}}} \ll 1 ; \\
S^{2} & =\frac{1}{6} \log \frac{27 U T}{2 l} \quad \text { if } \quad \log \bar{n}^{\frac{1}{2}} \gg 1, \quad \frac{3 U t_{0} / l}{\bar{n}^{\frac{1}{2}}\left(\log \bar{n}^{\frac{1}{2}}\right)^{\frac{1}{2}}} \gg 1 .
\end{aligned}
$$

For this case, $S^{2}$ is again an increasing function of $T$ provided $T$ is sufficiently small compared with $t_{0}$, but when $T$ is sufficiently large $S^{2}$ has a constant value independent of $T$.

It therefore appears that molecular diffusion is of great importance in determining the statistical properties of the longitudinal displacement however small it may be, provided it is not exactly zero and the value of $T$ is sufficiently large. It is to be emphasized, however, that the present theory is tentative because of the simplifying assumptions that have been made about the way in which molecular diffusion works, and it should really be regarded as no more than a first approximation. It should also be mentioned that the numerical coefficients in the above results are not expected to be exactly correct because the geometry of the model is obviously a rough approximation to that of a porous medium.

\section{Longitudinal dispersion}

The dispersion of a cloud of marked particles relative to its centre follows from the statistics of the displacement of a single particle only if the time or number of steps is large enough for the paths of the different particles to become statistically independent. As far as the longitudinal dispersion is concerned, there is no apparent reason why this should not be so after the particles have made 100 or so steps, provided the original dimensions of the cloud are large compared with a step length, although it is difficult to formulate satisfactory arguments to make this statement rigorous. It is therefore reasonable to assume that the above results for the longitudinal displacement may be applied to the dispersion of a cloud. A longitudinal diffusivity, $\kappa_{l}$ say, may be defined by the equation

$$
\kappa_{l}=\frac{1}{2 T} \overline{(X-U T)^{2}}=\frac{1}{2} U l S^{2},
$$

where $S^{2}$ is given by (4.3) or (4.4) as the case may be. The dimensionless ratio $U l / \kappa_{l}$ is not a constant but depends upon $t_{0}, t_{1}, T$ and $l / U$. The longitudinal dispersion does not satisfy the ordinary diffusion equation unless $T$ is sufficiently large for $(4.3 a)$ or $(4.4 a)$ to apply, since otherwise the diffusivity is a function of $T$.

* The result (4.4b) is essentially that given by Josselin de Jong (1958). 
It seems worth while mentioning here one case of physical interest for which the result (4.4b) may possibly apply for all $T$, i.e. for which the dispersion may be calculated on the assumptions that all marked particles move with the average speed in the pore and that the duration of a step may be unbounded. Consider the displacement of a fluid in a porous medium by another fluid of identical viscosity (and density if hydrostatic pressures are important), and suppose that the two fluids do not mix. If the fluids are homogeneous, molecular diffusion does not enter the problem. When the interfacial tension is sufficiently large for the ratio of $\mu U$ to the interfacial tension to be small compared with one, then to a good approximation the meniscus between the two fluids in a pore will extend across the pore and move with a velocity equal to that of the average speed through the pore.* Assuming that the presence of the menisci does not significantly affect the flow field through the porous medium, the relative motion of the two fluids may be calculated on the assumption that the meniscus does not exist and every fluid particle moves with the average speed in the pore, and the distribution of relative concentration in the mixing region should therefore be asymptotically normal with a variance given by $(4.4 b)$, with $t_{0}=\infty$, for all sufficiently large $T$.

\section{Lateral dispersion}

It follows from the assumption of the statistical independence of the paths of different particles that the transverse or lateral dispersion may be described by an effective diffusivity, $\kappa_{l}$ say, defined by

$$
\kappa_{l}=\frac{1}{2 T} \bar{Y}^{2}=\frac{3}{16} U l,
$$

from (4.2). The lateral dispersion is therefore independent of molecular diffusion and the dimensionless number $U l / \kappa_{\ell}$ is constant.

There is, however, considerable doubt about the correctness of (4.6). Sir Geoffrey Taylor has pointed out that in an exactly two-dimensional flow through an exactly two-dimensional porous medium, the requirement that streamlines cannot cross implies that the width of a stream of marked fluid remains constant, variations of the order of the grain size being neglected. In other words, although the centre line of the stream may wander about from side to side with a mean square displacement given by a result similar to (4.2) (provided, of course, there are no rigid side walls) the paths of individual particles in the stream are so correlated that the width of the stream remains constant. Thus in two dimensions, either one or both are false of the assumptions that the paths of different fluid particles become statistically independent and that the lateral displacement in successive steps by a single particle are statistically uncorrelated. It is not obvious which of these assumptions is worse since the presence of rigid side walls parallel to the average velocity seems to make a difference. If side walls are there, the path of a single element is approximately a straight line, so there must be a high correlation between the lateral displacement in successive steps; if side walls are not there, there is no apparent reason why such a correlation should exist.

* This result was pointed out by Sir Geoffrey Taylor. 
The situation in three dimensions is even more obscure. Streamlines may now pass round one another, but it is unlikely that both of the assumptions just mentioned are valid unless some other effect is present to reduce the correlation, e.g. a complicated streamline pattern at the pore junctions which would have the effect of twisting streamlines around each other, or even some turbulence, although this is very unlikely to be present when the average motion satisfies Darcy's law. There seems to be hardly any experimental evidence on the lateral dispersion.

\section{Comparison between theory and experiment for the longitudinal dispersion}

The experiments of Von Rosenberg (1956). Von Rosenberg observed the width of the mixing region or concentration front between two miscible liquids of equal viscosities and densities (benzene and ethyl $n$-butyrate) after passage with either of three velocities through three packed towers, filled with Ottawa sand, of various lengths, the front being initially sharp. Table 1 gives the value of $l S^{2}$, defined as $(1 / U T)(\bar{X}-\overline{U T})^{2}$, calculated from the data on the assumption that the concentration curve is derived from a normal distribution.

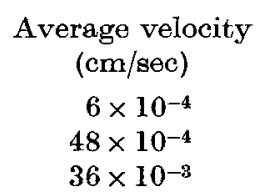
Average velocity
$(\mathrm{cm} / \mathrm{sec})$
$48 \times 10^{-1}$
$36 \times 10^{-3}$

\begin{tabular}{ccc}
\multicolumn{3}{c}{ Length of tower (cm) } \\
\hline 27.6 & 57.9 & 118.0 \\
0.085 & 0.075 & 0.058 \\
0.085 & 0.110 & 0.110 \\
0.078 & 0.128 & 0.158
\end{tabular}

TABLE 1. Values of $l S^{2}$ in $\mathrm{cm}$ calculated from the data of Von Rosenberg (1956). Each figure is the result of a separate experiment.

Exact quantitative comparison is not possible because Von Rosenberg does not give the average grain size of the sand. However, let us for the sake of example suppose that $l=0 \cdot 1 \mathrm{~cm}$ (which seems reasonable though possibly a little large) and $\kappa=1.5 \times 10^{-5} \mathrm{~cm}^{2} / \mathrm{sec}$ (this is of the same order of magnitude as the diffusivities of simple organic liquids in benzene); then $t_{0}=330 \mathrm{sec}$. The values of $l / U$ are 170,21 and $3 \mathrm{sec}$, respectively, and the theory given here will therefore not apply to the experiments with the smallest velocity since for these $U t_{0} / l$ is not large compared with unity. If we suppose further that $a / l=\frac{1}{5}$ (the value which seems appropriate to glass beads and which will suffice here as an estimate) then $t_{1}=3 \mathrm{sec}$, so that, for the purposes of rough comparison at least, the appropriate theoretical results are those of (4.4). On examining the relative values of $U t_{0} / l$ and $\bar{n}$, we find that all the experiments with $U=48 \times 10^{-4} \mathrm{~cm} / \mathrm{sec}$ satisfy the conditions of $(4.4 a)$, according to which $S^{2}$ is independent of $T$ and therefore of the length of the tower; whereas those with $U=36 \times 10^{-3} \mathrm{~cm} / \mathrm{sec}$ are intermediate between the conditions of $(4.4 a)$ and $(4.4 b)$ with a tendency to satisfy the latter, rather than the former, according to which $S^{2}$ is a logarithmic function of the length of the tower. It is clear from table 1 that there is qualitative agreement between the theory and experiment. As regards quantitative agreement, it 
follows from (4.4a) that the value of $l S^{2}$ for $U=48 \times 10^{-4} \mathrm{~cm} / \mathrm{sec}$ is $0.12 \mathrm{~cm}$; and from $(4.4 b)$ the values of $l S^{2}$ are $0 \cdot 13,0 \cdot 14,0 \cdot 16 \mathrm{~cm}$, respectively, for the three tower lengths. The quantitative agreement would be improved by taking a slightly smaller value of $l$.

The experiments of Ebach \& White (1958). In these, the dispersion was measured of Pontomine sky blue $6 \mathrm{BX}$ dye in water flowing through a $5 \cdot 12 \mathrm{ft}$. long tower packed with glass beads of diameter 0.0083 in. For those experiments in which the Reynolds number based on the average velocity and bead diameter was less than one (so that Darcy's law may be satisfied), the observed values of $U l / \kappa_{l}$, with $l$ equal to the bead diameter, were scattered between 0.4 and 0.6 with no systematic variation for 9 values of $U$ between 0.00077 and $0.0315 \mathrm{ft}$. $/ \mathrm{sec}$.

With a value of $\kappa=10^{-6} \mathrm{~cm}^{2} / \mathrm{sec}$ (which seems appropriate for an organic dye), the value of $t_{0}$ is $220 \mathrm{sec}$ and that of $t_{1}$ is $2 \cdot 2 \mathrm{sec}$, taking $a / l=\frac{1}{5}$. The value of $l / U$ lies between $0.9 \mathrm{sec}$ and $0.02 \mathrm{sec}$ and the appropriate theoretical results are those of (4.3). The value of $\bar{n}$ is the same for all the experiments and is $1 \cdot 1 \times 10^{4}$.

Comparison of the values of $U t_{0} / l, U t_{1} / l$ and $\bar{n}$ shows that for the smallest velocities the conditions $(4.3 b)$ are satisfied, whereas for the largest velocities the conditions (4.3c) are satisfied. According to (4.3b) the value of $S^{2}$ increases from $2 \cdot 8$ to 3.7 as the velocity goes from $0.00077 \mathrm{ft}$. $/ \mathrm{sec}$ to $0.0315 \mathrm{ft}$. $/ \mathrm{sec}$, and according to $(4.3 c)$ the value of $S^{2}$ is $3 \cdot 4$ for all velocities. Thus, according to the theory $S^{2}$ should go from $2 \cdot 8$ to $3 \cdot 4$ as the velocity goes from the smallest value to the largest, and the value of $U l / \kappa_{l}=2 / S^{2}$ goes from 0.72 to 0.59 . These results are in good agreement with the experimental observations bearing in mind the large experimental scatter.

The experiments of Josselin de Jong (1958). In these the width of a concentration front, which was initially sharp, between water and salt solution was measured after passage through a tower of glass beads of diameter $0.02 \mathrm{~cm}$ after distances of $2 \cdot 5,7 \cdot 5,12.5$ and $17.5 \mathrm{~cm}$. The value of $U$ was $0.92 \times 10^{-2} \mathrm{~cm} / \mathrm{sec}$. Josselin de Jong compared the observed values with the predictions of $(4.4 b)$ and found approximate agreement if the value of $l$ was taken as $0.008 \mathrm{~cm}$, i.e. about onethird the bead diameter.

Comparing his results with the theory of the present paper, we have $t_{0}=13 \mathrm{sec}$ with $\kappa=1.5 \times 10^{-5} \mathrm{~cm}^{2} / \mathrm{sec}$ and $l=0.02 \mathrm{~cm}$, and $t_{1}=0.13 \mathrm{sec}$ taking $a / l=\frac{1}{5}$. The value of $l / U$ is $2 \cdot 2 \mathrm{sec}$, so the appropriate theoretical results are (4.4). Further, the values of $\bar{n}$ are $190,560,940$ and 1320, and comparison with (4.4) shows that it is the conditions of $(4.4 a)$ which appear to be satisfied, and $(4.4 b)$ does not appear to be valid.

Now from $(4.4 a)$, the value of $\left(2 \kappa_{l} T / X\right)^{\frac{1}{2}}=l^{\frac{1}{2}} S$ is $0 \cdot 13 \mathrm{~cm}^{\frac{1}{2}}, l$ being taken as the diameter of the beads $0.02 \mathrm{~cm}$, and the value of this quantity according to the experimental data is approximately $0 \cdot 14$, so that the agreement with $(4.4 a)$ is better than that with $(4.4 b)$, in accordance with the present theory.

To sum up, the agreement between the present theory and the three sets of experimental data is encouraging, but a more thorough experimental investigation is clearly required. 


\section{Remarks on the dispersion when Darcy's law not satisfied}

An essential feature of the model put forward in $\S 2$ is that the velocity of the fluid through a pore should be proportional to the pressure drop between the ends of the pore. This is so if the inertia of the fluid is negligible and the flow in the pores is domir ated by viscosity, and it was pointed out in $\$ 2$ that this is equivalent to assuming that the flow obeys Darcy's law, equation (2.3), and that the streamline pattern is independent of the average velocity. For sufficiently large values of the velocity, or more precisely of the Reynolds number based on the pore length, the average velocity and the kinematic velocity, the fluid inertia is not negligible, Darcy's law is not satisfied, and there is no reason to expect that the dispersion is described by the results of the previous section. Since this case obtains in many practical applications, it seems worth while examining our model to see if by means of suitable modifications predictions can be made about the dispersion at high values of the average velocity.

The difficulty is that the flow is then much more complicated and the calculation of the velocity field in the pores becomes troublesome. Also, the assumption that the pores are straight and uniform becomes suspect because the curvature of the streamlines is important when the fluid inertia is not negligible. However, the dispersion is primarily a kinematic, as opposed to a dynamic, property of the flow field, and there is no obvious reason why the path of a marked particle should not be approximated to by a random walk of randomly orientated straight steps of random duration. But in order to calculate the duration of the steps it is necessary to consider the dynamics of the flow, and it has not proved possible so far to do this in a satisfactory manner.

It seems worth while to examine qualitatively the model consisting of an assembly of straight uniform pores as the Reynolds number increases. It follows from dimensional analysis that

$$
\frac{\kappa_{l}}{U l}=F\left(\frac{U T}{l}, \frac{U l}{\kappa}, \frac{U l}{\nu}\right)
$$

( $\nu$ denotes the kinematic viscosity), where $F$ is independent of the Reynolds number $U l / \nu$ when it is not too large (the value of $U l / \nu$ for which departures from Darcy's law become significant varies greatly from one type of porous medium to another and will be anywhere between 10 and 100). Now as the Reynolds number increases, the velocity profile will cease to be parabolic all along the pore and will be flat at the ends, i.e. the extent of the inlet regions will increase with the Reynolds number. Thus one effect of increasing $U l / \nu$ is to make the velocity of the fluid in a pore more uniform and hence to decrease the dispersion, until the limit is reached in which the velocity is uniform throughout the pore except for thin layers near the pore walls. It does not follow immediately that $F$ decreases as $U l / \nu$ increases because other Reynolds number effects are present; in particular, the ratio of the velocity in a pore to the average velocity of the fluid through the medium will be Reynolds number dependent. However, the longitudinal dispersion was calculated using a random walk model which takes this effect into account and it appears that the longitudinal dispersion again decreases with 
increase of Reynolds number, so that the indications are that $F$ is a decreasing function of $U l / v$.

This random walk model is rather crude, but the results are not without interest and it seems worth while placing them on record. With the same notation as used in $\S 2$, it is supposed that the average speed in a pore is proportional, owing to the effects of inertia, to some power of the pressure difference between the ends of the pore, and further that the pressure in the medium is $P^{\prime} x$. Accordingly, $\hat{q}=K P^{\prime} s \cos ^{s} \theta\left(0 \leqslant \theta \leqslant \frac{1}{2} \pi\right)$, where $s$ is a function of the Reynolds number and $K$ has the same value for every pore.

By means of the arguments of $\S 2$, the (spatial) average of velocity is

$$
U=\int_{0}^{2 \pi} \int_{0}^{\frac{1}{2} \pi} \frac{1}{2 \pi} \hat{q} \cos \theta \sin \theta d \theta d \phi=\frac{K P^{\prime s}}{s+2},
$$

and hence

$$
\hat{q}=(s+2) U \cos ^{s} \theta .
$$

It follows from (5.2) that the filter velocity is proportional to a power of the mean pressure gradient, and it is indeed found that this is sometimes so in practice (see Scheidegger 1957). A means of giving a value to $s$ is thereby afforded, and $s$ is usually between $\frac{1}{2}$ and 1 . We should intuitively expect $s$ to approach one when $U l / \nu$ is small, and to approach $\frac{1}{2}$ when the Reynolds number is large enough for the flow in the pores to be turbulent and the pressure drop to be proportional to the square of the velocity, and that in general $s$ decreases as $U l / \nu$ increases.

The duration of a step is

$$
t=\frac{l}{\hat{q}}=\frac{l}{(s+2) U \cos ^{s} \theta},
$$

if it is supposed that the velocity profile in a pore is flat and that all fluid particles, except for a negligible fraction, move with the average speed $\hat{q}$. The probability of a given step occurring may then be calculated as in $\$ 2$ and we have

$$
d \mathbf{P}=\frac{s+1}{2 \pi} \sin \theta \cos ^{s} \theta d \theta d \phi \quad\left(0 \leqslant \theta \leqslant \frac{1}{2} \pi, 0 \leqslant \phi<2 \pi\right) .
$$

The (ensemble average) variance of $t$ now exists and it is not necessary to bring in molecular diffusion.

The statistical properties of the displacement of a single particle may now be calculated exactly as in $\S 3.1$ to $§ 3.5$, apart from changes in numerical values. The results are, with the same notation as employed previously:

$$
\begin{gathered}
\bar{Y}=\bar{Z}=0 ; \\
\bar{n}=\frac{s+2}{s+1} \frac{U T}{l}, \quad \bar{X}=U T, \quad \text { if } \quad \bar{n} \gg 1 ;
\end{gathered}
$$

the lateral displacement is normally distributed with

$$
\bar{Y}^{2}=\bar{Z}^{2}=\frac{s+2}{(s+1)(s+3)} U l T \quad \text { if } \quad \bar{n}^{\frac{1}{2}} \gg 1
$$

and $(X-U T) / T^{\frac{1}{2}}$ is normally distributed with

$$
\frac{1}{T} \overline{(X-U T)^{2}}=\frac{(s+1)^{2}}{(1-s)(s+2)(s+3)} U l \quad \text { if } \quad \bar{n}^{\frac{I}{(2}(1-s) / s} \gg 1 .
$$


(This last condition on $\bar{n}$ requires that $s$ is not too close to 1 and follows from an analysis similar to that carried out in $\$ 3.7$. If it is not satisfied and $(1-s) \log \bar{n} \ll 1$, then it may be shown that the result (4.4b) applies, as is to be expected since when $s \rightarrow 1$ the duration of a step as given by (5.4) tends to the value used in calculating $(4.4 b)$.)

It follows from the assumption that the paths of different particles become statistically independent that

and

$$
\begin{aligned}
\frac{\kappa_{t}}{U l} & =\frac{s+2}{2(s+1)(s+3)}, \\
\frac{\kappa_{l}}{U l} & =\frac{(s+1)^{2}}{2(1-s)(s+2)(s+3)},
\end{aligned}
$$

where $\kappa_{b}$ and $\kappa_{l}$ denote the effective lateral and longitudinal diffusivities, respectively. It will be noted that (5.11) decreases as $s$ decreases from 1 to $\frac{1}{2}$.

The values of $\kappa_{l} / U l$ reported in the literature for high Reynolds numbers show considerable scatter but seem to lie between 0.5 and 1 . The value of $(5.11)$ is 0.76 when $s=0.8$.

The values of $\kappa_{t} / O l$ given by (5.10) seem to be a little high, but this discrepancy may well be explained by the falseness of the assumption that the lateral displacement in successive steps are uncorrelated. As regards the variation of $\kappa_{l} / U l$ with Reynolds number, it seems that the main effect of increasing Reynolds number may be to decrease the correlation between the lateral displacement in successive steps, because of an increase in complexity of the streamline pattern at the pore junctions or because of some turbulent mixing taking place, and thus to increase the lateral dispersion.

I wish to thank Sir Geoffrey Taylor for suggesting this investigation and for the benefit of several discussions; also Dr G. K. Batchelor for constructive criticism of an earlier draft.

\section{Appendix}

Integrals of the form

$$
I=\int f(\cos \theta) g\left\{\left(1+p_{1}\right) \cos \theta+p_{2} \sin \theta \cos \phi+p_{3} \sin \theta \sin \phi\right\} \sin \theta
$$

over the domain

$$
\times \exp \left[-\lambda\left(p_{1}^{2}+p_{2}^{2}+p_{3}^{2}\right)\right] d \theta d \phi d p_{1} d p_{2} d p_{3}
$$

$$
0<\theta<\pi, \quad 0<\phi<2 \pi, \quad\left(1+p_{1}\right) \cos \theta+p_{2} \sin \theta \cos \phi+p_{3} \sin \theta \sin \phi>0,
$$

occur in $\S 2$. The method of evaluation consists in making the transformation

$$
\begin{aligned}
& p_{1}^{\prime}=p_{1} \cos \theta+p_{2} \sin \theta \cos \phi+p_{3} \sin \theta \sin \phi, \\
& p_{2}^{\prime}=-p_{1} \sin \theta+p_{2} \cos \theta \cos \phi+p_{3} \cos \theta \sin \phi, \\
& p_{3}^{\prime}=-p_{2} \sin \phi+p_{3} \cos \phi,
\end{aligned}
$$


which gives

$$
\begin{array}{r}
I=\int_{\substack{0<\theta<\pi \\
0<\phi<2 \pi \\
\cos \theta+p_{1}^{\prime}>0}} f(\cos \theta) g\left(\cos \theta+p_{1}^{\prime}\right) \sin \theta \exp \left[-\lambda\left(p_{1}^{2}+p_{2}^{2}+p_{3}^{2}\right)\right] d \theta d \phi d p_{1}^{\prime} d p_{2}^{\prime} d p_{3}^{\prime} \\
=-\frac{2 \pi^{2}}{\lambda} \iint_{\substack{0<\theta<\pi \\
p+\cos \theta>0}} f(\cos \theta) g(p+\cos \theta) d p d(\cos \theta)
\end{array}
$$

on integration with respect to $p_{2}^{\prime}, p_{3}^{\prime}$ and $\phi$, and writing $p_{1}^{\prime}=p$. The evaluation of the last integral is straightforward for the cases which occur in $\S 2$.

\section{REFERENCES}

ARIs, R. 1956 Proc. Roy. Soc. A, 235, 67.

BARON, T. 1952 Chem. Eng. Progr. 48, 118.

Cramér, H. 1946 Mathematical Methods of Statistics. Princeton University Press.

EвaCh, E. A. \& White, R. R. 1958 J. Amer. Inst. Chem. Engrs, 4, 61.

JefFreys, H. \& Jeffreys, B. S. 1950 Methods of Mathematical Physics. 2nd ed. Cambridge Univorsity Press.

Josselin DE JoNG, G. DE 1958 Trans. Amer. Geophys. Un. 39, 67.

MoHenry, K. W. \& Wirhelum, R. H. 1957 J. Amer. Inst. Chem. Engrs, 3, 83.

Scheidegger, A. E. $1954 J$. Appl. Phys. 25, 994.

SCheidegger, A. E. 1957 The Physics of Flow through Porous Media. Toronto University Press.

TAYlor, G. I. 1953 Proc. Roy. Soc. A, 219, 186.

TAYlor, G. I. 1954 Proc. Roy. Soc. A, 225, 473.

Von Rosenberg, D. V. 1956 J. Amer. Inst. Chem. Engrs, 2, 55. 